Title:

\title{
Two independent modes of chromosome organization are revealed by cohesin removal
}

Authors:

Wibke Schwarzer ${ }^{1, *}$, Nezar Abdennur ${ }^{2, *}$, Anton Goloborodko ${ }^{3, *}$, Aleksandra Pekowska ${ }^{4}$, Geoffrey Fudenberg ${ }^{5}$, Yann Loe-Mie ${ }^{6,7}$, Nuno A Fonseca ${ }^{8}$, Wolfgang Huber ${ }^{4}$, Christian Haering ${ }^{9}$, Leonid Mirny ${ }^{3,5} \#$, Francois Spitz ${ }^{1.4,6,7} \#$

Affiliations:

${ }^{1}$ Developmental Biology Unit. European Molecular Biology Laboratory. 69117 Heidelberg, Germany

${ }^{2}$ Computational and Systems Biology Program, MIT, Cambridge, USA

${ }^{3}$ Department of Physics, MIT, Cambridge, USA

${ }^{4}$ Genome Biology Unit. European Molecular Biology Laboratory. 69117 Heidelberg, Germany

${ }^{5}$ Institute for Medical Engineering and Sciences, MIT, Cambridge, USA

${ }^{6}$ Institut Pasteur, (Epi)genomics of Animal Development Unit, Developmental and Stem Cell Biology Department. Institut Pasteur. 75015 Paris, France

${ }^{7}$ CNRS, UMR3738, 25 rue du Dr Roux, 75015 Paris, France

${ }^{8}$ European Bioinformatics Institute. European Molecular Biology Laboratory. Wellcome Trust Genome Campus, Hinxton, UK.

9 Cell Biology and Biophysics Unit. European Molecular Biology Department. 69117 Heidelberg, Germany

* co-first authors

\# co-corresponding authors: francois.spitz@pasteur.fr - leonid@mit.edu 
The three-dimensional organization of chromosomes is tightly related to their biological function ${ }^{1}$. Both imaging and chromosome conformation capture studies have revealed several layers of organization ${ }^{2-4}$ : segregation into active and inactive compartments at the megabase scale ${ }^{5}$, and partitioning into domains (TADs) ${ }^{6,7}$ and associated loops ${ }^{8}$ at the sub-megabase scale. Yet, it remains unclear how these layers of genome organization form, interact with one another, and contribute to or result from genome activities. TADs seem to have critical roles in regulating gene expression by promoting or preventing interactions between promoters and distant cis-acting regulatory elements ${ }^{9-14}$, and different architectural proteins, including cohesin, have been proposed to play central roles in their formation ${ }^{15,16}$. However, experimental depletions of these proteins have resulted in marginal changes in chromosome organization ${ }^{17-19}$. Here, we show that deletion of the cohesin-loading factor, $\mathrm{Nipbl}$, leads to loss of chromosome-associated cohesin and results in dramatic genome reorganization. TADs and associated loops vanish globally, even in the absence of transcriptional changes. In contrast, segregation into compartments is preserved and even reinforced. Strikingly, the disappearance of TADs unmasks a finer compartment structure that accurately reflects the underlying epigenetic landscape. These observations demonstrate that the 3D organization of the genome results from the independent action of two distinct mechanisms: 1) cohesin-independent segregation of the genome into fine-scale compartment regions, defined by the underlying chromatin state; and 2) cohesindependent formation of TADs possibly by the recently proposed loop extrusion mechanism 20,21, enabling long-range and target-specific activity of promiscuous enhancers. The interplay between these mechanisms creates an architecture that is more complex than a simple hierarchy of layers and can be central to guiding normal development. 
Different architectural protein complexes have been proposed to contribute to interphase chromosomal organization, notably the site-specific DNA-binding factor CTCF and cohesin, which co-localize at TAD boundaries and loops ${ }^{6,8}$ 22,23. Mutations of different cohesin subunits in humans lead to different disorders including Cornelia de Lange syndrome ${ }^{24}$, which are proposed to result in part from gene mis-expression due to defective chromatin folding. While recent models and experimental evidence suggest that regions bound by CTCF and cohesin contribute to organizing loops and TAD boundaries ${ }^{8,9,20,25,26} \cdot 27$, experimental depletion of cohesin subunits or CTCF have shown a limited impact on local chromatin interactions, with most structures, including TADs, appearing largely unaffected ${ }^{17-19}$. Therefore, it remains unclear to what extent these complexes play a part in the genome's 3D organization.

Here, instead of depleting cohesin's constitutive subunits, we interfered with the loading of cohesin complexes by deleting the loading factor $N I P B L / S C C 2$. Our reasoning was that since the turnover of chromosome-bound cohesin is relatively fast in interphase (in the range of few minutes) ${ }^{28,29}$, constant loading is required for its presence on DNA. Efficient deletion of Nipbl in non-dividing hepatocytes was achieved by using a liver-specific, tamoxifen-inducible Cre driver (Fig.1a). This conditional, inducible approach allowed us to circumvent the lethality of

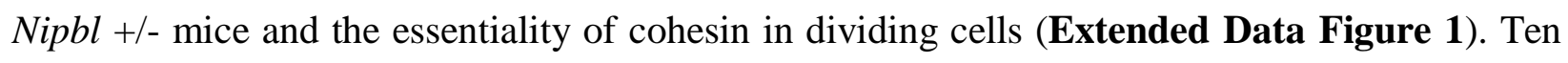
days after tamoxifen injection, Nipbl expression was dramatically reduced (Fig.1b) and led to a displacement of cohesin proteins from the chromatin fraction to the soluble nuclear fraction, indicating a strong eviction of cohesin from chromosomes (Fig.1c). No particular pathological signs compared to control animals (either mock-injected Nipblflox/flox; App-Cre animals or tamoxifen-injected Nipbl+/+; App-Cre animals) were observed in the liver, and hepatocytes showed no sign of either cell death or proliferation (Extended Data Figure 1).

To assess the consequence of Nipbl depletion and cohesin unloading on chromosome organization, we performed tethered chromatin conformation capture (TCC, referred below as Hi-C) ${ }^{30}$ on purified hepatocytes from wildtype (WT), tamoxifen control (TAM) and $\triangle \mathrm{Nipbl}$ animals (Fig.1d). For each of these three conditions, two biological replicates were generated. The contact maps obtained from each biological replicate showed extensive similarities (Extended Data Figure 2). For further analyses, we pooled the two replicate datasets to generate Hi-C maps for the three different conditions. In parallel, we determined the complete 
transcriptome of WT, TAM and $\triangle \mathrm{Nipbl}$ hepatocytes by strand-specific RNA-seq, and performed ChIP-seq for $\mathrm{H} 3 \mathrm{~K} 4 \mathrm{me} 3$ and $\mathrm{H} 3 \mathrm{~K} 27 \mathrm{ac}$ to compare the regulatory programs of the different cell populations (see Supplemental Methods). We compare Hi-C maps for $\Delta \mathrm{Nipbl}$ and control cells at each characterized level of chromatin organization (Extended Data Figure 3): compartments, TADs, loops, and global scaling of the contact probability $P(s)$ with genomic separation $s^{31}$.

While previous studies using cohesin depletion observed very mild effects on TADs (Extended Data Figure 4), our Hi-C data reveal a striking effect of Nipbl deletion on genome organization (Fig.1d). Compared to WT and TAM control samples, $\triangle$ Nipbl cells show a genome-wide disappearance of TADs, leaving genome compartmentalization relatively unaffected (Fig.1d). Disappearance of TADs in $\triangle \mathrm{Nipbl}$ is widespread and evident in individual maps (Fig.2a), as well as on the composite map constructed by averaging the $\Delta \mathrm{Nipbl} \mathrm{Hi}-\mathrm{C}$ map around locations of TAD boundaries detected in WT maps (Fig2b, Extended Data Figure 5). While in regions of inactive chromatin no other structures emerge upon loss of TADs, some local organisation is retained in active and repressed regions (Extended Data Figure 5, Supplemental Methods). As shown below, these structures reflect fine A/B compartmentalization of the genome, rather than residual TADs.

Loops between specific elements within TADs, which are visible as bright corners in about 50\% of TADs ${ }^{8}$, disappear in $\triangle \mathrm{Nipbl}$ maps too, showing up to 4 fold reduction in contact frequency (Fig.2c and Extended Data Figures 2c and 4). Similarly, the insulation and directionality of the contact footprint of CTCF sites disappear upon Nipbl deletion as seen in composite maps centered at oriented CTCF sites (Extended Data Figure 6). Importantly, these structural changes cannot be attributed to altered expression in $\triangle \mathrm{Nipbl}$ cells, as TADs vanished even in regions with unchanged expression (Fig.2d) as well as in regions with up- and down-regulated transcription (Extended Data Figure 5c). This major reorganization of chromatin architecture is also reflected in the curves of contact frequency $P(s)$ as a function of genomic distance $s^{4,32}$. In WT or TAM samples, like in other mammalian cells ${ }^{20,21}$, the scaling of $P(s)$ has two different regimes: a more shallow decay for $\mathrm{s}<200 \mathrm{~Kb}\left(P(s) \sim s^{-0.7}\right)$, and a steeper scaling for $200 \mathrm{~Kb}<\mathrm{s}<$ $3 \mathrm{Mb}\left(P(s) \sim s^{-1.2}\right)$. We find that the loss of chromatin-associated cohesin in $\Delta \mathrm{Nipbl}$ cells leads to disappearance of the first regime, producing a single decay of contact probability across the whole range (Fig.2e). This observation suggests that the first scaling regime reflects the 
compaction of the genome into TADs. We confirmed this by separately calculating $P(s)$ within and between TADs: in WT and TAM cells, $P(s)$ within TADs decreases more slowly than $\mathrm{P}(\mathrm{s})$ for loci separated by a TAD boundary (between TADs). In $\triangle \mathrm{Nipbl,} \mathrm{the} \mathrm{difference} \mathrm{between} \mathrm{these}$ two curves is greatly reduced, indicating that the insulating effect of TAD boundaries have diminished and that chromatin folding is more uniform and decondensed (Fig.2e), which is consistent with decondensation observed by imaging upon Nipbl reduction ${ }^{33}$.

TAD formation has been proposed to result from a cohesin-dependent loop extrusion mechanism, where chromatin-associated cohesins extrude progressively expanding loops, until becoming stalled by boundary elements, e.g. bound and properly oriented CTCF ${ }^{20,21}$ (Fig.2f). Each TAD is a collection of dynamic intra-TAD loops that shows $P(s) \sim s^{-0.7}$ intra-TAD scaling. To test whether loop extrusion ${ }^{20,21}$ can reproduce our experimental findings we simulated the effects of Nipbl depletion in a 400kb TAD by reducing the number of loop-extruding cohesins. For each extruding cohesin concentration, we calculated simulated Hi-C maps and $P(s)$ within and between TADs. In agreement with our experimental data, 8-fold depletion leads to (i) noticeable disappearance of TADs and loops; (ii) loss of $P(s) \sim s^{-0.7}$ regime in the scaling; (iii) decompaction of the chromatin (Fig.2g). Interestingly, TADs were still pronounced at 2-fold cohesin depletion, as has been observed in earlier studies ${ }^{17-19}$, demonstrating that near-complete removal of chromatin-associated cohesins is necessary for observing a complete disappearance of TADs and loops. Together, these analyses and the observed effects of Nipbl deletion indicate that cohesin plays a central role in the compaction of chromosomes into TADs. Moreover, they support the role of cohesin as a loop extruding factor that forms TADs and loops in interphase 20,21 .

We next examined the A/B-compartmentalization of chromatin in $\Delta \mathrm{Nipbl}$ cells. Surprisingly, unlike the drastic loss of TADs, compartmentalization still exists in $\Delta \mathrm{Nipbl}$ cells (Extended Data Figure 7) and, in fact, the segregation of A-type and B-type compartmental regions increased ( 1.8 fold between the most A- and B-like loci) (Extended Data Figure 8). Closer examination of Hi-C data and compartment tracks, however, reveals widespread local alteration of genome compartmentalization in $\Delta \mathrm{Nipbl}$ cells, with formation of smaller compartmental structures (Fig.3a, Extended Data Figure 9). We found that this persistent compartmentalization of the $\Delta \mathrm{Nipbl}$ genome explains most of the remaining or new domains and 
boundaries seen in $\Delta \mathrm{Nipbl} \mathrm{Hi}-\mathrm{C}$ maps (Extended Data Figures 9-10). The finer compartmentalization is reflected in the shorter autocorrelation length of the compartment track ( $150 \mathrm{~Kb}$ in $\triangle \mathrm{Nipbl} v \mathrm{vs} \sim 500 \mathrm{~Kb}$ in $\mathrm{WT}$ and TAM (Extended Data Figure 11), and in the emergence of short B-like compartment regions inside A-regions (Extended Data Figure 11). These emerging B-like regions possess the hallmarks of compartmentalization: (i) they are visible as local depressions in the $\Delta \mathrm{Nipbl}$ compartment track (Fig.3b) and (ii) they show preferential interactions with other B-regions both in far cis- and in trans-chromosomal maps (Fig.3b). Consequently, they do not represent newly formed TADs since TADs do not exhibit preferential long range interactions. In contrast, B-rich regions, despite a complete loss of TADs, do not experience a similar degree of fragmentation in $\Delta \mathrm{Nipbl}$ cells (Extended Data Figure 11b). Taken together, our observations defy the common notion of TADs simply being the building blocks of larger compartmental segments. Instead, TADs and compartments represent two independent types of chromosomal organization.

Next, we examined how the observed structural reorganization is related to functional characteristics of the genome. Strikingly, we found that the compartment structure of the $\Delta \mathrm{Nipbl}$ genome reflects local transcriptional activity and chromatin state better than the compartment structure of the WT (Fig.3c-e, Extended data Figure 12). The compartment track of $\Delta$ Nipbl cells shows a stronger correlation with $\Delta$ Nipbl tracks of activity-associated epigenetic marks, H3K27ac, H3K4me3, and gene expression, as well as related wildtype tracks, e.g. H3K27ac, H3K4me3, DNase hypersensitivity, TF binding etc., smoothed over a wide range of window sizes (Extended Data Figure 11c). To understand the relationship between epigenetic state and the change in compartment status, we compared the compartment tracks to the mouse liver chromatin state segmentation (ChromHMM ${ }^{34}$ ) simplified into three state categories: Active, Repressed and Inert (see Methods). While the compartmentalization of inert regions is relatively unaffected by $\mathrm{Nipbl}$ deletion, regions of repressed and active chromatin further diverge in their compartment status (Fig.3cd), producing local peaks in the compartment track in active regions, and local B-like depressions in repressed regions (Fig.3c). Furthermore, regions of facultative lamin-B1 association ${ }^{35,36}$ are enriched in regions showing a reduction in compartment signal (from A to B-like), while those showing lamin-B1 association across different mouse cell lines are primarily B-type in both WT and the mutant (Extended Data Figure 13, Methods). Importantly, these changes in compartmentalization cannot be attributed to changes in expression 
or in the activity marks (H3K27ac, $\mathrm{H} 3 \mathrm{~K} 4 \mathrm{me} 3)$, which are largely unperturbed in the mutant at the scales relevant to compartmentalization (Extended Data Figures 12,14-15). In summary, in the absence of chromosome-associated cohesin allowd the genome to adopt a compartmental organization that closely reflects the local functional state of the genome. Incidentally, the fact that the association between compartmental structure and epigenetic state is observed even when considering the wildtype epigenetic landscape suggests that the landscape is largely unaffected by loss of chromosome-associated cohesin (Fig.3e, Extended Data Figures 12,15).

Altogether, these results indicate that chromatin has an intrinsic tendency to form smallscale, specific compartments that reflect its local epigenetic landscape and transcriptional activity. However, in wild-type cells, this close association between epigenetic state and 3D organization is reduced, likely because the distinct loop-extruding activity of cohesin that leads to formation of TADs can bring together and mix loci with opposing states.

Deletion of Nipbl and the disappearance of TADs led to altered transcription. About a thousand genes are significantly mis-expressed (637 down-regulated, median fold-change $=0.32$, 487 up-regulated, median fold-change $=3.15$, with DESeq2 tools) upon Nipbl deletion and TAD disappearance (Fig.4a and Extended Data Figure 16). Importantly, while H3K27ac (and $\mathrm{H} 3 \mathrm{~K} 4 \mathrm{me} 3$ ) peaks at promoter regions of affected genes change in coherence with expression changes, distal peaks (marking active distant enhancers) are mostly unaffected (Extended Data Figure 17). This conservation of distal enhancer signal indicates that the regulatory potential of the cells was mostly unperturbed, yet transcriptional changes did occur. We noticed that downregulated genes are surrounded by a larger intergenic space (defined by the distance separating the TSS of their immediate neighbours) than up-regulated or unaffected ones (Extended Data Figure 16b) and transcriptional changes are concentrated within regions that normally form larger TADs. If there is no reliable way to identify a priori genes for which distal regulatory interactions are essential, this characteristic genomic context of transcriptional alterations is consistent with defective long-range regulatory interactions in $\Delta$ Nipbl cells. Remarkably, while we saw more genes down-regulated than up-regulated, we noticed an inverse phenomenon in intergenic regions, with widespread up-regulation of exo-genic (intergenic or antisense intragenic) transcription (Fig.4a). Using a conservative approach (see Methods), we found 1107 non-genic transcripts or transcribed regions, which showed at least an 8-fold enhanced 
transcription in $\Delta \mathrm{Nipbl}$ cells; amongst these, 232 correspond to new non-coding RNAs, which are not detected or barely detected in WT or TAM samples, and for most of them not annotated (Extended Data Figure 16d). The new transcription is often bi-directional, (Fig.4bc, Extended Data Figure 17) and occurs either at pre-existing - but often inactive - promoters (marked by small peaks of H3K4me3) or at enhancers (Fig.4d-e, Extended Data Figure 17). We saw several examples of reciprocal expression changes (i.e. down-regulation of a gene being followed by up-regulation of an adjacent gene or of a new non-coding transcripts) (Fig.4b, Extended Data Figure 17e), but often, new non-coding transcription arises without measurable impact on surrounding genes. While the chromatin profile suggests that enhancers retain their normal activity and therefore regulatory potential, this shift from gene-promoter transcription to intergenic transcription initiated in the vicinity of distal regulatory elements suggests that the deletion of Nipbl and resulting absence of TADs and loops impairs enhancer communication: with a reduced range of contact, some enhancers cannot reach their target promoters and transfer their activity on nearby alternative, sometimes cryptic, targets (including themselves).

Overall, our findings provide new important insights into the mechanisms that organize the genome's 3D structures and their relation to gene expression. Our data show that local chromatin structures result from the overlapping action of two totally independent mechanisms (Fig.4f). This overlap challenges the standard hierarchical, representation of genome architecture in which individual TADs (or small contact domains) combine to form active (A) or inactive (B) compartmental regions. Partitioning of the genome into these active and inactive compartmental regions and their preferential self-interaction are achieved by a cohesin-independent mechanism. This mechanism acts pervasively, across scales, and notably at a much smaller scale than previously appreciated, reflecting local transcriptional activity and the epigenetic landscape. This fine compartment structure is however blurred by the action of a second, cohesin-dependent mechanism, which compacts chromatin locally, independently of its status, but is constrained by CTCF-enriched boundary elements ${ }^{20,21}$. This picture is consistent with the formation of dynamic cis-loops by a cohesin-dependent loop extrusion process. Our genome-wide data provide support to the notion that cohesin-dependent cis-looping and resulting TADs allow for communication between distal regulatory regions in a reliable and tuneable manner ${ }^{14}$, while the activitydependent compartmentalization may subsequently reinforce and maintain these interactions. 
Mammalian genomic contact maps, revealed by Hi-C, result from the superimposition of these two distinct mechanisms. Their relative contributions can vary between loci, and, for a given locus, between cell types. The co-existence of two processes with different modes and scales of action - together with the intrinsic disorder of chromosome conformations - can explain the difficulties in the field in delineating and unambiguously classifying the different population-averaged patterns seen in Hi-C maps and described as "domains" (TADs ${ }^{6,7}$, $\operatorname{subTADs}^{15}$, metaTADs ${ }^{37}$, physical domains ${ }^{38}$, contact domains ${ }^{8}$ etc.), which have led to discussions regarding their existence as structural or functional entities. The experimental ability to now distinguish these two modes of chromosome organization demonstrates that a distinct cohesin-dependent biological mechanism gives rise to the local contact domains identifiable as TADs in Hi-C maps and provides avenues to dissect the process(es) governing their formation and maintenance, as well as to distinguish cause from consequence in the relationship between gene expression and chromatin folding. 


\section{Acknowledgments}

This work would not have been possible without the important contribution of the members of the EMBL Laboratory Animal Resources Facility, particularly Silke Feller, for animal welfare and husbandry and of the EMBL Genomics Core Facility for sequencing the different genomic libraries. We thank members of the Mirny and the Spitz labs, John Marioni (EMBL/EBI) and Heather Marlow (IP) for many productive discussions and helpful suggestions. We are grateful to Hugo B. Brandão for providing a MATLAB-based visualization tool. We thank Ana Losada (CNIO) for generously providing antibodies. W.S and A.P were supported by an EMBL Interdisciplinary Postdoc (EIPOD) Fellowship under Marie Curie Actions COFUND. The work in the Mirny lab is supported by R01 GM114190, U54 DK107980 from the National Institute of Health, and 1504942 from the National Science Foundation. The collaboration is also partially supported by MIT-France MISTI Fund. The work in the Spitz lab was supported by the European Molecular Biology Laboratory, the Pasteur Institute and the Deutsche Forschungsgesellschaft (DFG grant: SP 1331/3-1). Funding from the European Commission's Seventh Framework Programme through the Collaborative Research Project RADIANT (Grant Agreement no: 305626, to W.H.) contributed also to this work.

\section{Author Contributions}

W.S. and F.S. conceived the study and designed the experiments, with input and advice from C.H. W.S. performed all the experiments with the help of A.P for TCC, who also carried out preliminary analyses of the TCC datasets, with advice from W.H.. N.A. and A.G. performed computational analysis of Hi-C, RNA-seq, ChIP-seq and other relevant datasets. Y.L-M and N.A.F. contributed to analysis of transcription data. G.F. performed computer simulations of cohesin depletion and assisted with data analysis and paper writing. L.M. provided advice on data analysis and simulations. W.S., N.A., A.G., L.M. and F.S. wrote the paper with input from the other authors.

\section{Author Information.}

The authors declare no competing financial interests. Correspondence and requests for materials should be addressed to F.S. (francois.spitz@ pasteur.fr) and to L.M. (leonid@ mit.edu). 
bioRxiv preprint doi: https://doi.org/10.1101/094185; this version posted December 15,2016 . The copyright holder for this preprint (which was not certified by peer review) is the author/funder, who has granted bioRxiv a license to display the preprint in perpetuity. It is made available under aCC-BY 4.0 International license. 


\section{References}

1. Bickmore, W. A. \& van Steensel, B. Genome Architecture: Domain Organization of Interphase Chromosomes. Cell 152, 1270-1284 (2013).

2. Gibcus, J. H. \& Dekker, J. The Hierarchy of the 3D Genome. Mol Cell 49, 773-782 (2013).

3. Dixon, J. R., Gorkin, D. U. \& Ren, B. Chromatin Domains: The Unit of Chromosome Organization. Mol Cell 62, 668-680 (2016).

4. Dekker, J. \& Mirny, L. The 3D Genome as Moderator of Chromosomal Communication. Cell 164, 1110-1121 (2016).

5. Boettiger, A. N. et al. Super-resolution imaging reveals distinct chromatin folding for different epigenetic states. Nature 529, 418-422 (2016).

6. Dixon, J. R. et al. Topological domains in mammalian genomes identified by analysis of chromatin interactions. Nature 485, 376-380 (2012).

7. Nora, E. P. et al. Spatial partitioning of the regulatory landscape of the X-inactivation centre. Nature 485, 381-385 (2012).

8. Rao, S. S. P. et al. A 3D Map of the Human Genome at Kilobase Resolution Reveals Principles of Chromatin Looping. Cell (2014). doi:10.1016/j.cell.2014.11.021

9. Lupiáñez, D. G. et al. Disruptions of topological chromatin domains cause pathogenic rewiring of gene-enhancer interactions. Cell 161, 1012-1025 (2015).

10. Tsujimura, T. et al. A discrete transition zone organizes the topological and regulatory autonomy of the adjacent tfap2c and bmp7 genes. PLoS Genet 11, e1004897 (2015).

11. Symmons, 0. et al. Functional and topological characteristics of mammalian regulatory domains. Genome Res 24, 390-400 (2014).

12. Nora, E. P., Dekker, J. \& Heard, E. Segmental folding of chromosomes: a basis for structural and regulatory chromosomal neighborhoods? Bioessays 35, 818-828 (2013).

13. Dowen, J. M. et al. Control of cell identity genes occurs in insulated neighborhoods in mammalian chromosomes. Cell 159, 374-387 (2014).

14. Symmons, 0. et al. The Shh Topological Domain Facilitates the Action of Remote Enhancers by Reducing the Effects of Genomic Distances. Dev Cell 39, 529-543 (2016).

15. Phillips-Cremins, J. E. et al. Architectural Protein Subclasses Shape 3D Organization of Genomes during Lineage Commitment. Cell 153, 1281-1295 (2013).

16. Merkenschlager, M. \& Odom, D. T. CTCF and cohesin: linking gene regulatory elements with their targets. Cell 152, 1285-1297 (2013).

17. Zuin, J. et al. Cohesin and CTCF differentially affect chromatin architecture and gene expression in human cells. Proc Natl Acad Sci U S A 111, 996-1001 (2014).

18. Sofueva, S. et al. Cohesin-mediated interactions organize chromosomal domain architecture. EMBO J 32, 3119-3129 (2013).

19. Seitan, V. C. et al. Cohesin-based chromatin interactions enable regulated gene expression within pre-existing architectural compartments. Genome Res 23, 2066-2077 (2013).

20. Sanborn, A. L. et al. Chromatin extrusion explains key features of loop and domain formation in wild-type and engineered genomes. Proc Natl Acad Sci U S A 112, E6456-E6465 (2015).

21. Fudenberg, G. et al. Formation of Chromosomal Domains by Loop Extrusion. Cell Reports 15, 2038-2049 (2016).

22. Wendt, K. et al. Cohesin mediates transcriptional insulation by CCCTC-binding factor. Nature (2008). doi:10.1038/nature06634

23. Parelho, V. et al. Cohesins Functionally Associate with CTCF on Mammalian Chromosome Arms. Cell (2008). doi:10.1016/j.cell.2008.01.011

24. Remeseiro, S., Cuadrado, A. \& Losada, A. Cohesin in development and disease. Development 140, 3715-3718 (2013). 
25. Guo, Y. et al. CRISPR Inversion of CTCF Sites Alters Genome Topology and Enhancer/Promoter Function. Cell 162, 900-910 (2015).

26. de Wit, E. et al. CTCF Binding Polarity Determines Chromatin Looping. Mol Cell (2015). doi:10.1016/j.molcel.2015.09.023

27. Vietri Rudan, M. et al. Comparative Hi-C Reveals that CTCF Underlies Evolution of Chromosomal Domain Architecture. Cell Reports 10,1297-1309 (2015).

28. Tedeschi, A. et al. Wapl is an essential regulator of chromatin structure and chromosome segregation. Nature 501, 564-568 (2013).

29. Gerlich, D., Koch, B., Dupeux, F., Peters, J.-M. \& Ellenberg, J. Live-cell imaging reveals a stable cohesin-chromatin interaction after but not before DNA replication. Curr Biol 16, 1571-1578 (2006).

30. Kalhor, R., Tjong, H., Jayathilaka, N., Alber, F. \& Chen, L. Genome architectures revealed by tethered chromosome conformation capture and population-based modeling. Nat Biotechnol 30, 90-98 (2012).

31. Lajoie, B. R., Dekker, J. \& Kaplan, N. The Hitchhiker's guide to Hi-C analysis: practical guidelines. Methods 72, 65-75 (2015).

32. Naumova, N. et al. Organization of the Mitotic Chromosome. Science (2013). doi:10.1126/science.1236083

33. Nolen, L. D., Boyle, S., Ansari, M., Pritchard, E. \& Bickmore, W. A. Regional chromatin decompaction in Cornelia de Lange syndrome associated with NIPBL disruption can be uncoupled from cohesin and CTCF. Hum Mol Genet (2013). doi:10.1093/hmg/ddt265

34. Ernst, J. et al. Mapping and analysis of chromatin state dynamics in nine human cell types. Nature 473, 43-49 (2011).

35. Peric-Hupkes, D. et al. Molecular maps of the reorganization of genome-nuclear lamina interactions during differentiation. Mol Cell 38, 603-613 (2010).

36. Meuleman, W. et al. Constitutive nuclear lamina-genome interactions are highly conserved and associated with A/T-rich sequence. Genome Res 23, 270-280 (2013).

37. Fraser, J. et al. Hierarchical folding and reorganization of chromosomes are linked to transcriptional changes in cellular differentiation. Mol Syst Biol 11, 852 (2015).

38. Sexton, T. et al. Three-dimensional folding and functional organization principles of the Drosophila genome. Cell 148, 458-472 (2012). 


\section{Figure 1}

a
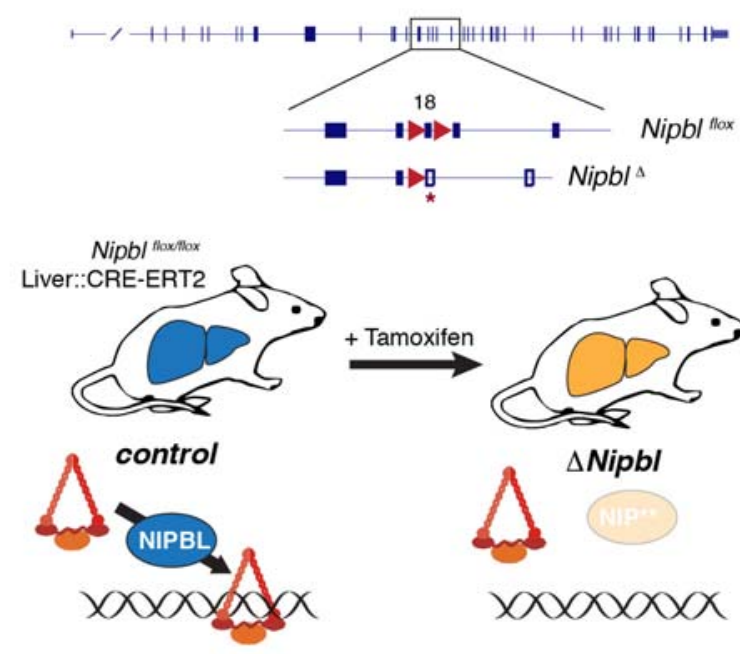

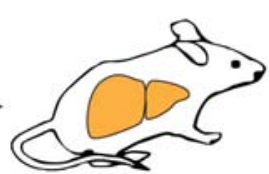

$\triangle$ Nipbl

A

$\infty \times 1 \times \infty \times$ b

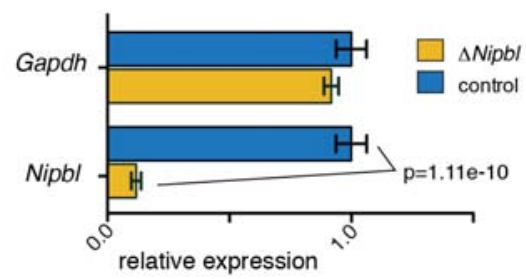

C

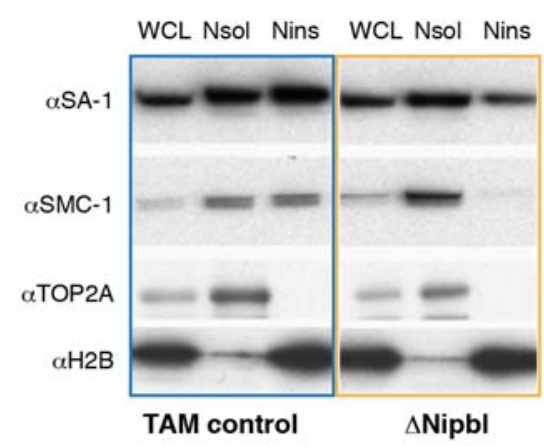

d

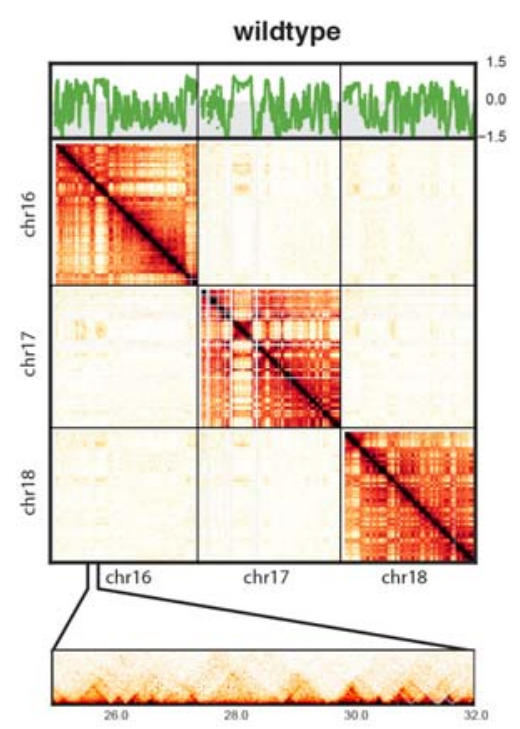

TAM

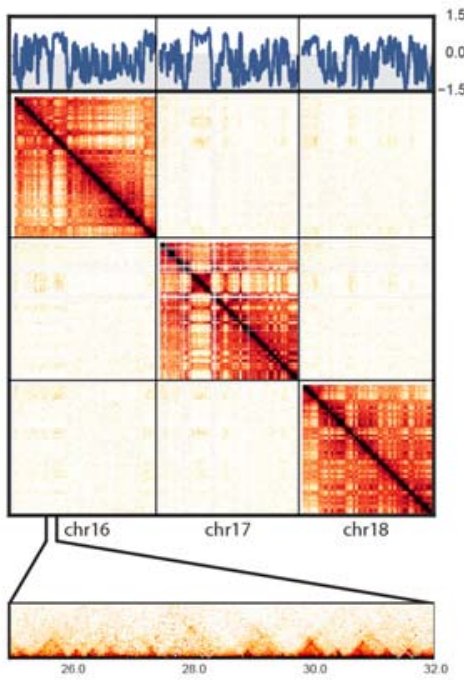

$\triangle$ NIPBL

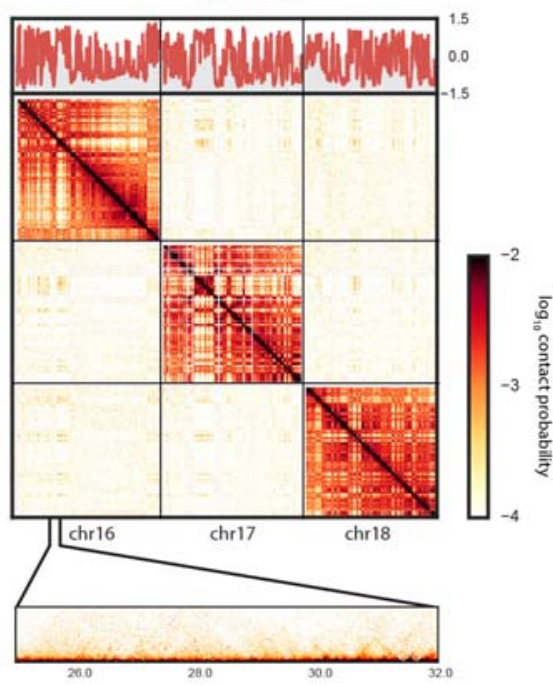

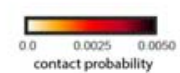

Figure 1. Overview of the experimental design. (a) A conditional Nipbl allele was created by flanking exon 18 with loxP sites. Upon CRE-mediated recombination, this exon is deleted leading to a frameshift and production of a non-functional protein (Fig. S1). A liver-specific driver for the conditional CRE-ERT2 fusion protein allows precise control of the deletion of $\mathrm{Niphl}$, which occurs in adult hepatocytes only after injection of Tamoxifen. In absence of Nipbl, 
cohesin (represented by a triangular ring) is not loaded on chromatin. (b) Expression level of Nipbl and Gapdh (control) by RT-qPCR in control $(\mathrm{n}=4)$ and $\Delta \mathrm{Nipbl}(\mathrm{n}=5)$ hepatocytes. Mean normalized gene expression (using Pgkl as internal control, and with expression level in WT set as 1) is displayed as mean and s.e.m. Statistical difference was assessed with unpaired $t$-test.(c) Western blots of hepatocytes protein extracts (WCL: whole cell lysate, Nsol (nuclear extract, soluble fraction) Nins (insoluble, chromatin fraction of nuclear extract)) showed displacement of cohesin structural subunits (SA-1, SMC1) from the chromatin-bound fraction. The efficient separation of the two fractions is shown by the distribution of the TOP2Aand H2B proteins (d-e) $\mathrm{Hi}-\mathrm{C}$ contact maps at $20 \mathrm{~kb}$ resolution of WT (left), TAM control (middle) and $\triangle \mathrm{Nipbl}$ cells (right). Top - compartment tracks calculated via eigenvector decomposition of intrachromosomal contact matrices. Middle - cis and trans contact maps of chr16-18. (e) an example of short-range contact patterns in the region chr16:25-32Mb. 
bioRxiv preprint doi: https://doi.org/10.1101/094185; this version posted December 15,2016 . The copyright holder for this preprint (which was not certified by peer review) is the author/funder, who has granted bioRxiv a license to display the preprint in perpetuity. It is made available under aCC-BY 4.0 International license.

\section{Figure 2}
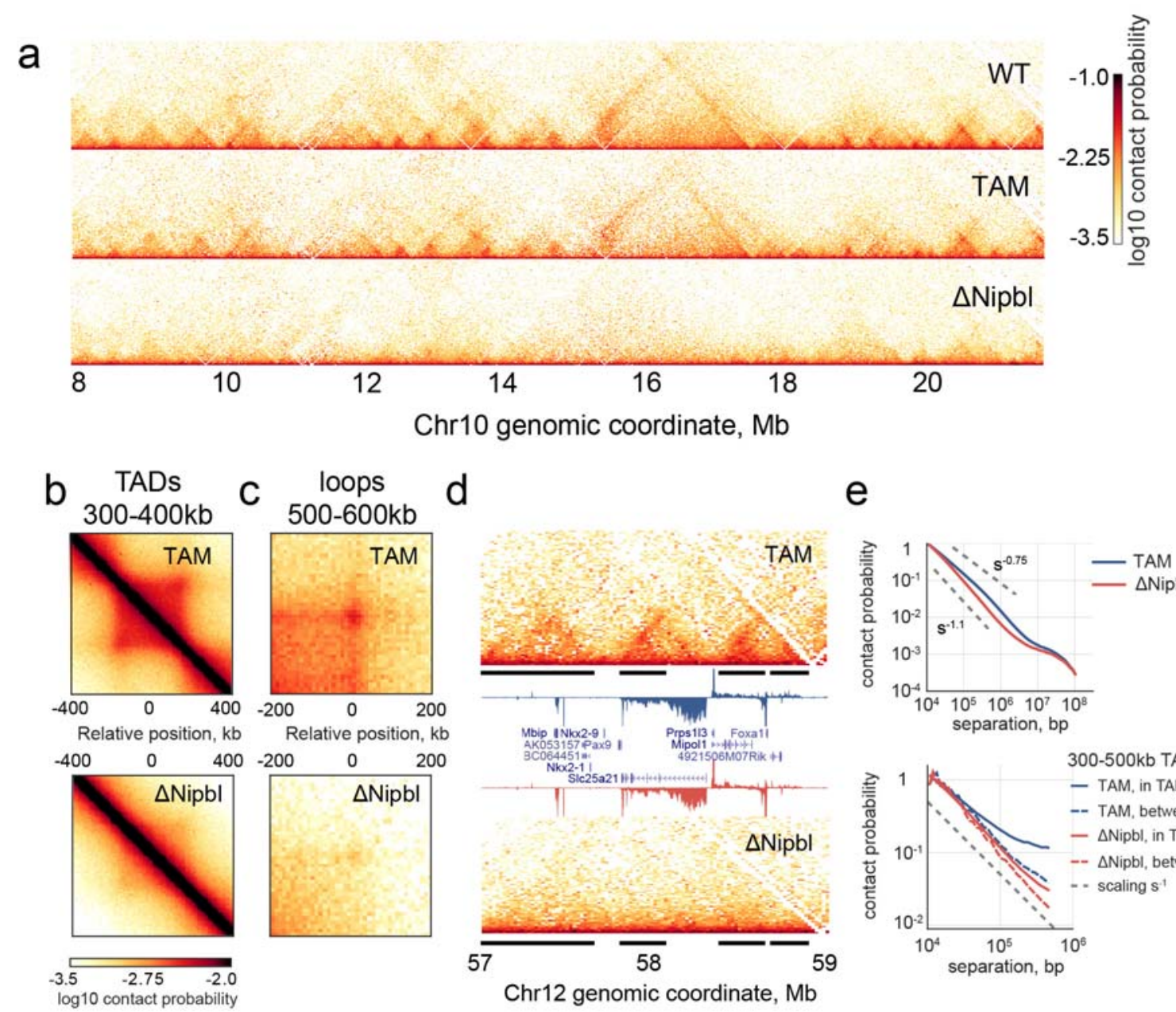

e

$\log 10$ contact probability

Chr12 genomic coordinate, $\mathrm{Mb}$
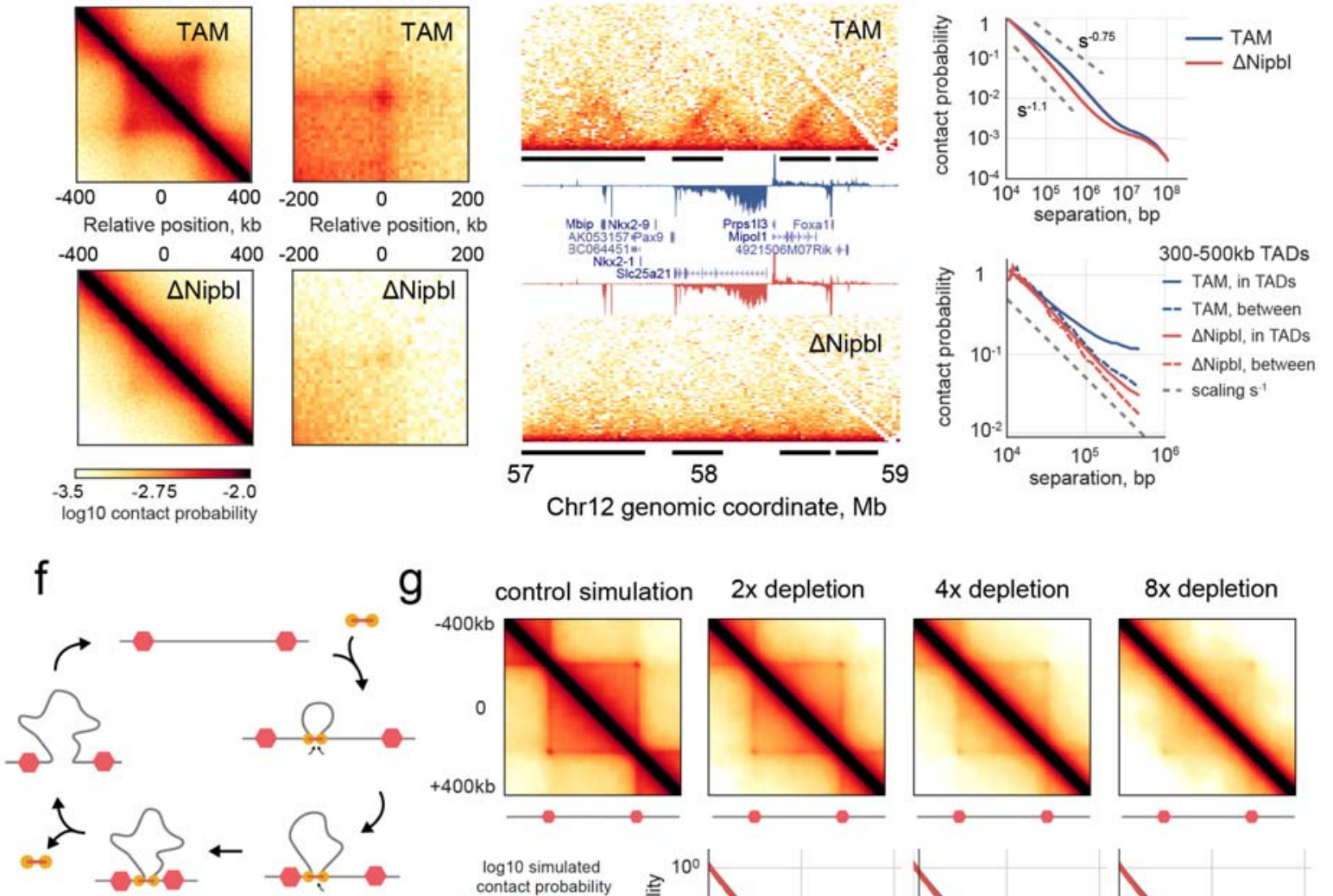

$8 x$ depletion

loop extruder boundary element

(cohesin)

(e.g. CTCF)

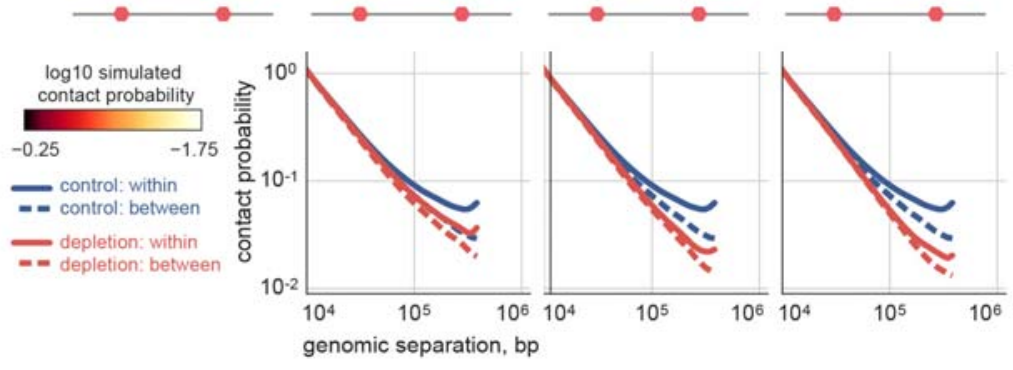


Figure 2. Nipbl deletion leads to disappearance of TADs and loops from Hi-C contact maps.

(a) The short-range contact map for chr10:8-21Mb illustrating robust uniform disappearance of TADs and loops. (b) The average map of 564 TADs of length $300-400 \mathrm{~kb}$ and (c) the average map of 102 loops of size 500-600kb. (d) The contact map of an example 2Mb region chr12:57$59 \mathrm{Mb}$ with overlaid expression track. Top and bottom panels $-\mathrm{Hi}-\mathrm{C}$ contact maps in TAM control and $\triangle \mathrm{Nipbl}$, correspondingly, black bars show TADs detected in WT contact maps. Middle panel - location of annotated genes and RPM normalized RNA-seq tracks in sense (above the axis) and anti-sense (below) directions, TAM in blue, $\triangle \mathrm{Nipbl}$ in red. (e) Top genome-wide curves of contact frequency $\mathrm{P}(\mathrm{s})$ vs genomic distance s in TAM control and $\Delta \mathrm{Nipbl}$, normalized to unity at $\mathrm{s}=10 \mathrm{~kb}$. Bottom $-\mathrm{P}(\mathrm{s})$ curves plotted separately for contacts formed within or between TADs of size $300-500 \mathrm{~kb}$. (f) The cartoon representation of the loop extrusion model of cohesin action, which explains how cohesins can form TADs and loops ${ }^{21}$. In this model, cohesins form cis-loops by first binding to adjacent loci on chromosomes (top and right diagrams). After binding, cohesins translocate along the fiber in both directions, effectively extruding a loop (bottom diagrams). Extrusion halts when cohesins reach boundary elements, formed either by a bound CTCF or any other impediment to extrusion. Extruded loops disassemble when cohesins unbind from the chromosome (left diagram). (g) Polymer simulations of loop extrusion reproduce the effects of cohesin depletion on Hi-C contact maps. Top row average maps of TADs formed on contact maps in polymer simulations of loop extrusion. Leftto-right: the impact of sequential cohesin depletion on the contact map of a TAD in simulations. Bottom row -contact frequency, $\mathrm{P}(\mathrm{s})$, versus genomic distance, s, calculated separately for contacts formed within and between TADs. 
bioRxiv preprint doi: https://doi.org/10.1101/094185; this version posted December 15,2016 . The copyright holder for this preprint (which was not certified by peer review) is the author/funder, who has granted bioRxiv a license to display the preprint in perpetuity. It is made available under aCC-BY 4.0 International license.

Figure 3

a

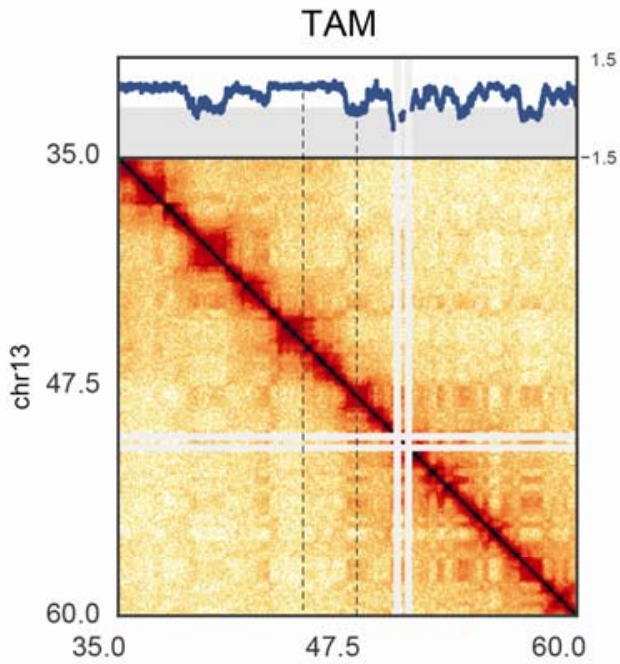

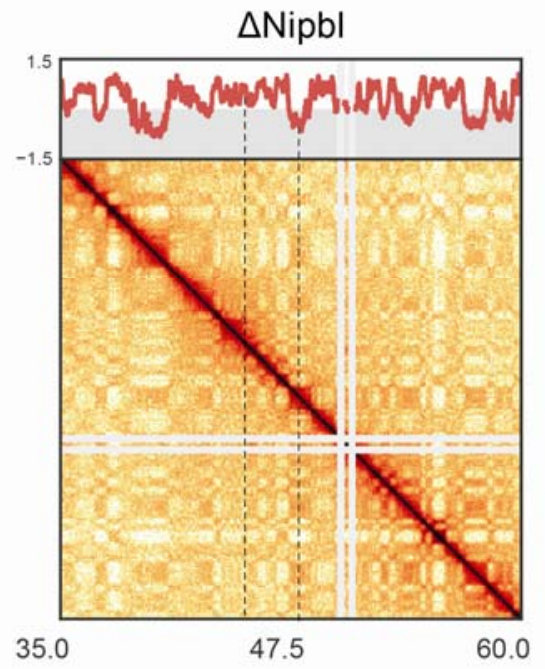

b
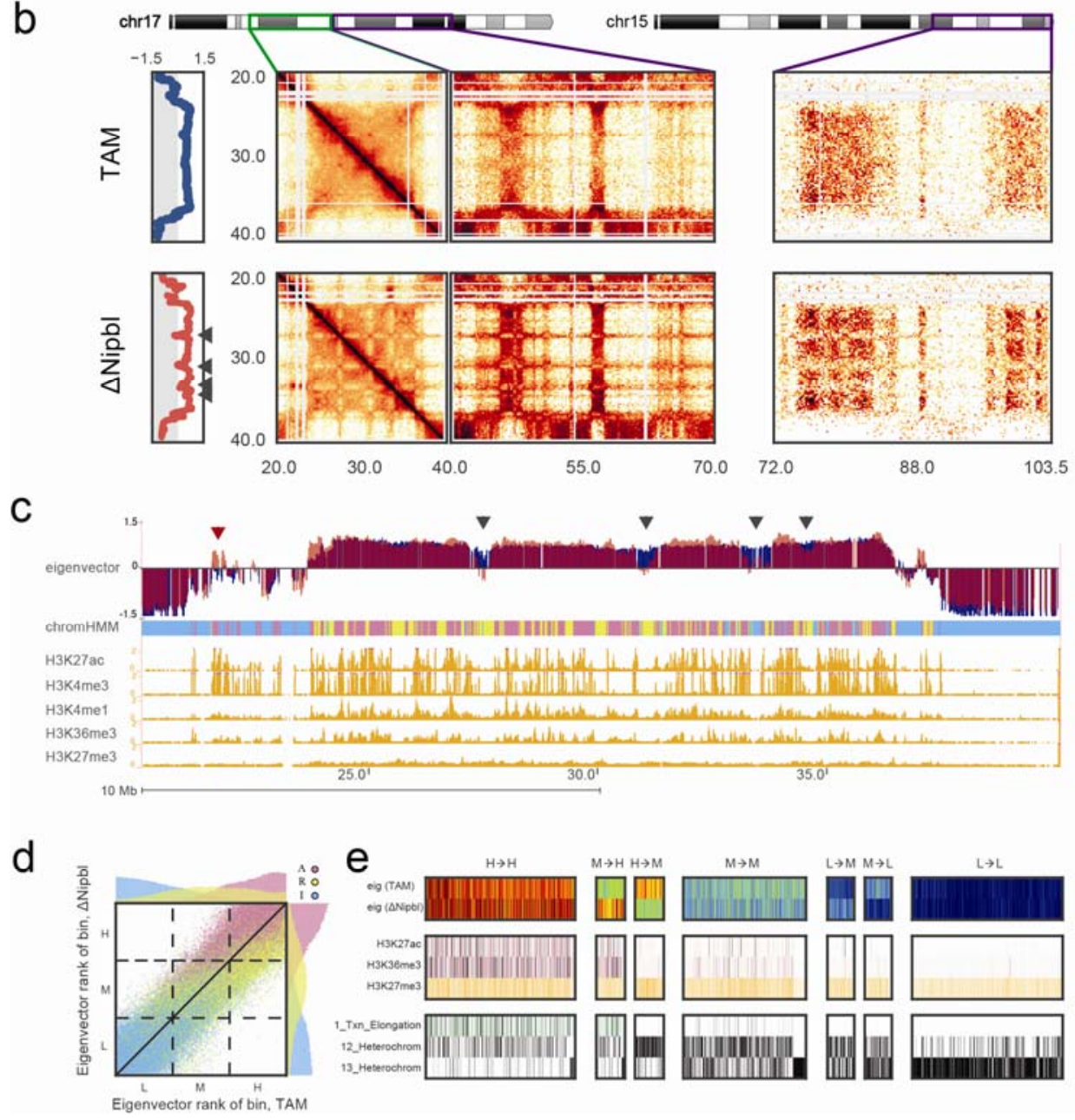


\section{Figure 3. Nipbl deletion leads to activity-dependent alteration of compartment structure.}

(a) An example region (chr13:35-60Mb) of a Hi-C contact map showing reorganization of compartment structure. Top panel - compartment signal calculated by eigenvector decomposition of the intra-chromosomal contact matrix at $20 \mathrm{~kb}$ resolution. Dashed lines indicate the smaller region displayed in Extended Data Figure 11. (b) An example of a large region with a uniformly positive (type A) compartment signal in TAM control cells (top) showing compartment fragmentation upon Nipbl deletion (bottom). Fragmentation is manifested in the alternating contact patterns of short- $(<10 \mathrm{Mb}$, middle left) and long-range cis (middle right) and trans contact maps (right panel) as well as in the eigenvector compartment signal. Arrows on the left panel indicate the regions of a local drop in compartment signal. (c) The loci experiencing a local drop (black arrowheads) in the compartment signal are depleted in epigenetic marks of activity. In contrast, active loci within B-rich regions can show a local increase in compartment signal (red arrowhead). Top to bottom: eigenvector compartment signal in TAM (blue) and $\Delta \mathrm{Nipbl}$ (red) cells; simplified ChromHMM state assignment of loci grouped into active (magenta) / repressed (yellow) / inert (cyan) states; ENCODE activity-related histone ChIP-seq for adult mouse liver cells. (d) Rank correlation of $20 \mathrm{~kb}$ resolution eigenvectors in TAM and $\triangle \mathrm{Nipbl}$, colored by simplified ChromHMM state. Top and right margins - histograms of eigenvector ranks split by simplified ChromHMM state in $\triangle \mathrm{Nipbl}$ (right) and TAM (top). The dashed lines show the tercile borders, splitting bins into equal-sized groups of low (L), middle (M) and high (H) eigenvector. (e) Epigenetic profiles of bins transitioning between eigenvector terciles upon Nibpl deletion. Top to bottom: eigenvector in WT and $\triangle$ Nipbl cells; ENCODE histone marks; ChromHMM states characteristic of active, repressed and inert chromatin. The bins that transitioned from the middle to the high tercile are enriched in activity marks, while bins transitioning from the high to the middle tercile were depleted in those marks. 


\section{Figure 4}

a

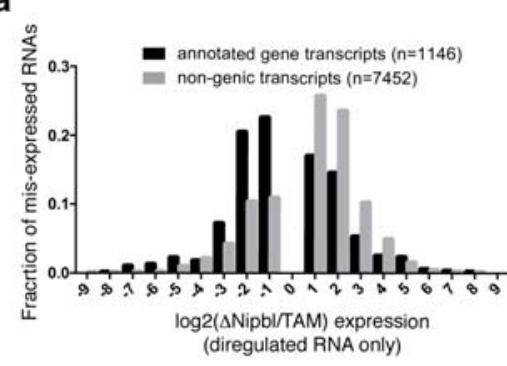

d

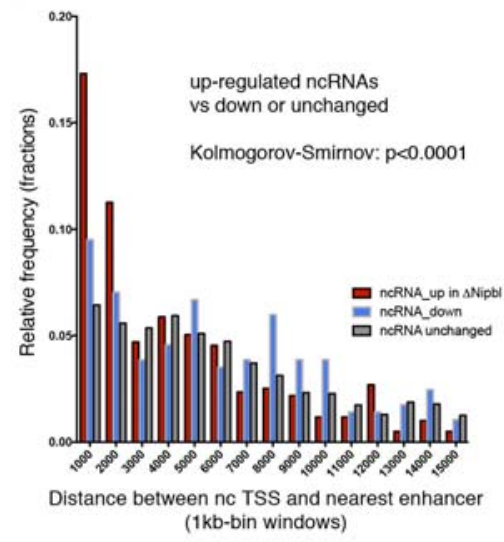

b

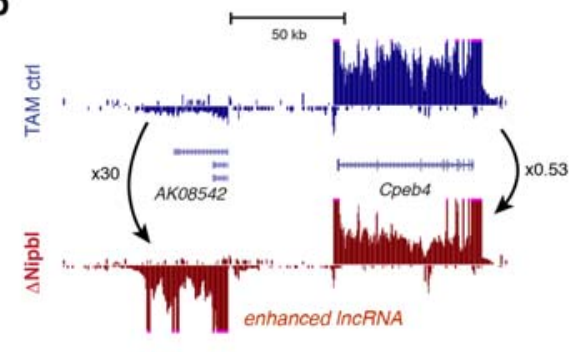

c

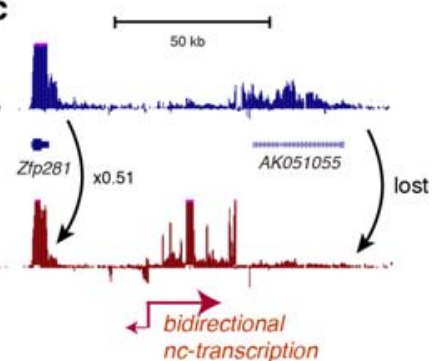

e
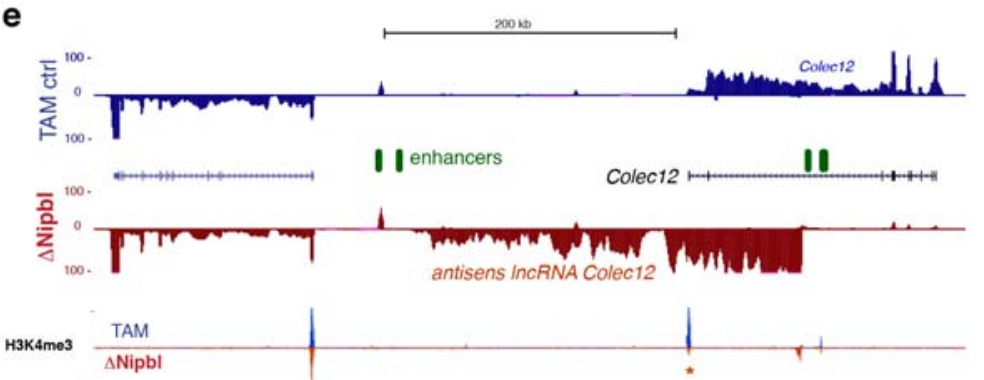

нзК27ac

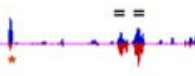

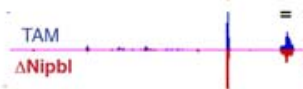

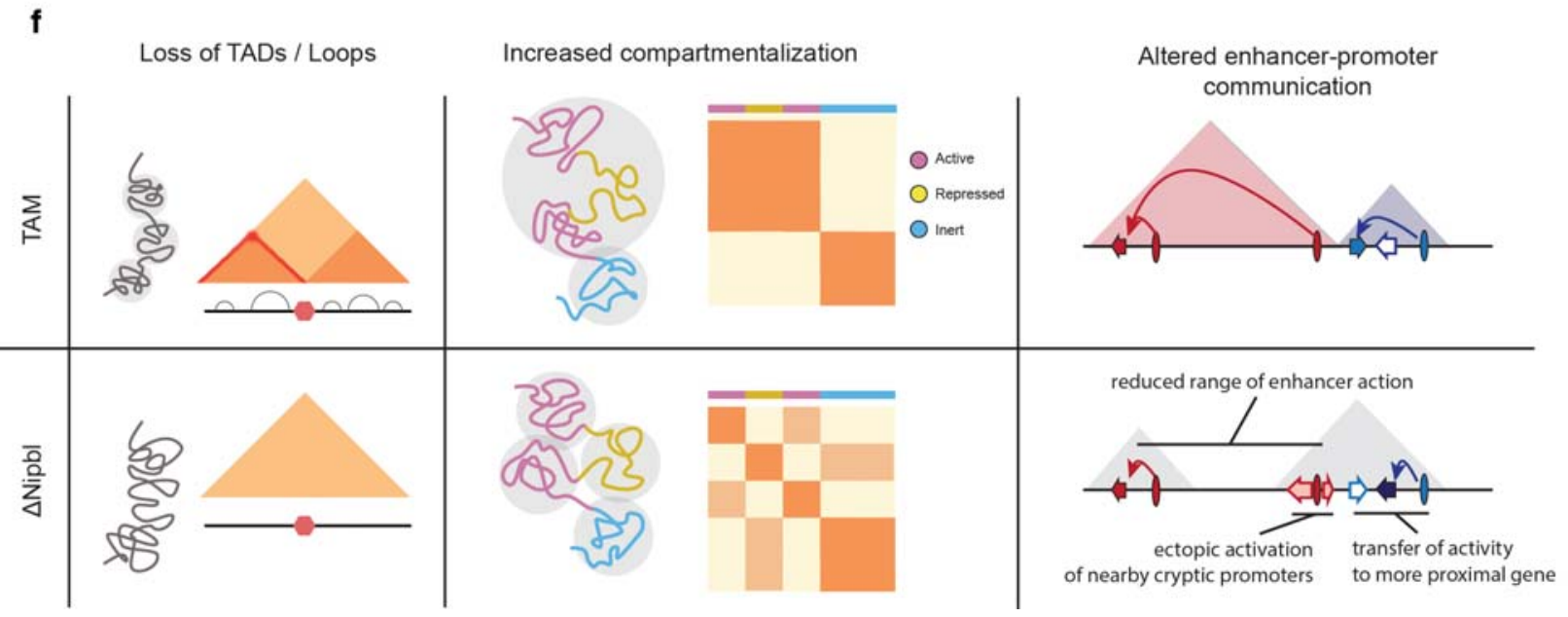

Figure 4. Transcriptional changes in Nipbl mutants reveal possible enhancer-promoter communication problems in absence of TADs. (a) Distribution of fold-changes for misexpressed genes (black) and exo-genic transcripts (light grey). Genes showing less than a twofold change in either direction were omitted. (b-c) Examples of transcriptional changes at different loci, with TAM controls stranded RNA-seq tracks in blue, and $\triangle$ Nipbl in red. The 
tracks combined data from four replicates for each condition. Transcriptional changes reported were observed in each replicate. (d) Distribution of distances between the transcriptional start of the ncRNAs and the nearest enhancer. (e) Transcriptional switch at the Colec12 locus. RNA-seq tracks show a disappearance of the Colec12 transcripts, replaced by an antisense transcript initiated from a intronic enhancer. $\mathrm{H} 3 \mathrm{~K} 4 \mathrm{me} 3$ and $\mathrm{H} 3 \mathrm{~K} 27 \mathrm{ac}$ profiles show no changes at distal enhancers (green ovals, marked by "=" on the tracks), while peaks at the Colec12 promoter disappear (red asterisk). (f) Summary of the results. TADs (coloured triangles) and loops disappear upon Nipbl deletion (left), unmasking a stronger and finer compartmentalization (middle) that is visible as a fragmented plaid pattern in the mutant Hi-C map relative to that of the wildtype and whose alternating member regions more faithfully track transcriptional activity. The resulting reduction of contact range (right) thwarts distant enhancers (ovals) from acting on their normal target genes (arrows, with coloured ones indicating active genes, white ones inactive), leading them to act instead on neighbouring genes or cryptic promoters located in their vicinity. The active units make up new compartmental regions (grey triangle). 


\section{EXTENDED DATA}

\section{Extended Data Figure 1}

a

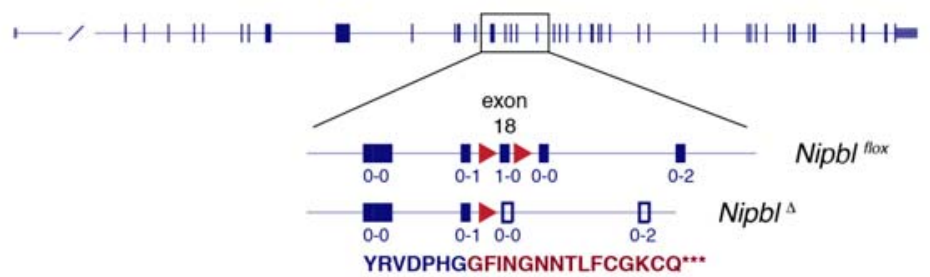

STOP in Nipb/s

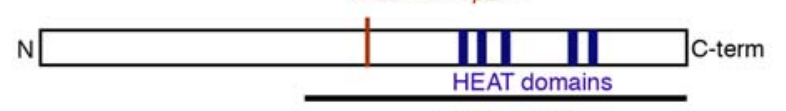

NIPBL/SCC2

conserved domain in bilateria

c

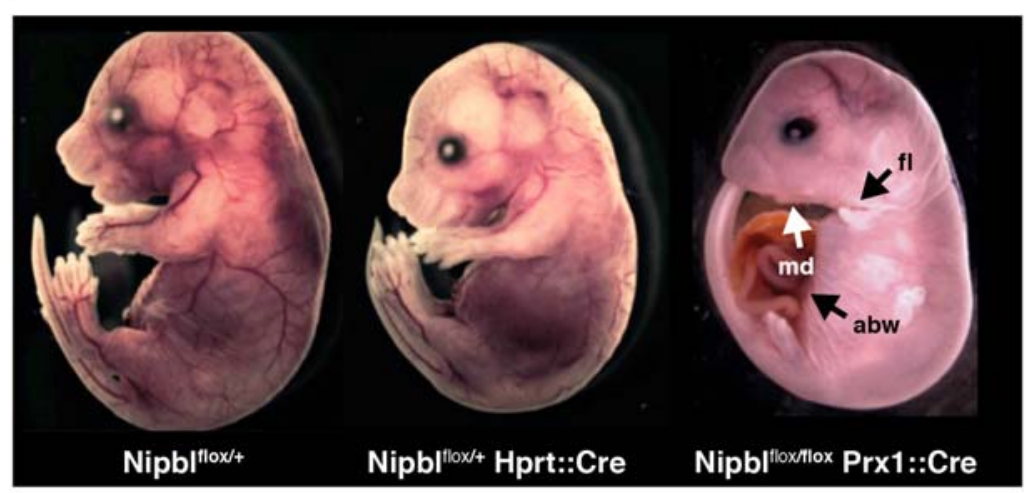

d

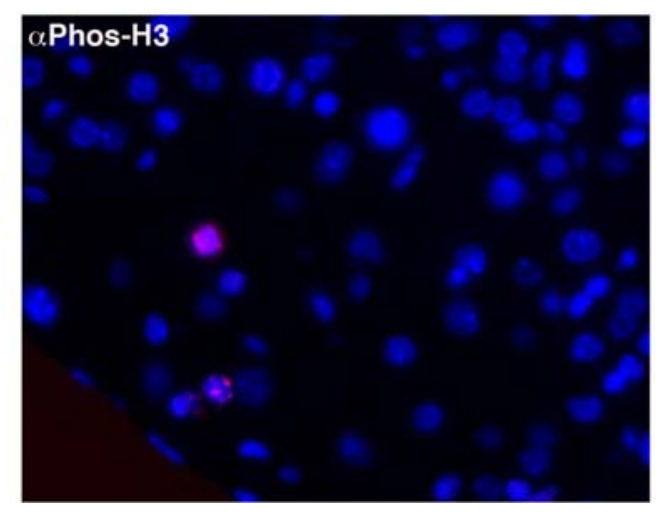

b
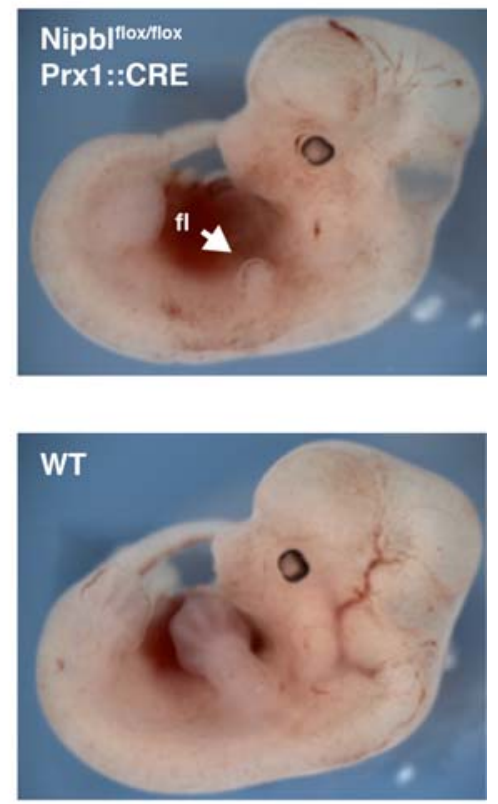

Extended Data Figure 1 - Conditional inactivation of Nipbl in mice. (a) Schematic representation of the conditional allele, with loxP sites (red triangles) flanking exon 18. The reading frame of each exon is indicated below the corresponding square, as " $\mathrm{x}-\mathrm{x}$ ". Deletion of 
exon 18 leads to a frame-shift introducing a premature stop codon (indicated by amino acids in red). The resulting protein lacks the critical HEAT domains conserved in NIPBL/SCC2 proteins. (b-c) E12 embryos (b) and E18 fetuses (c) carrying the conditional Nipbl allele (Nipbl flox $)$ and either ubiquitous (Hprt:Cre ${ }^{39}$ ) or limb-specific (Prx1::Cre ${ }^{40}$ ) Cre recombinase drivers. Structures expressing Cre are rapidly lost in Nipbl flox/flox animals. Heterozygous $N i p b l^{\text {flox/+ }}$ animals are grossly morphologically normal, but die soon after birth, as reported for other Nipbl loss of function alleles ${ }^{41}$. fl=forelimb; md:mandibule; abw=abdominal wall. (d-e) Histochemical staining of liver section of adult $\Delta$ Nipbl hepatocytes (Nipblflox/flox; Ttr::CreERT2; 10 days after Tamoxifen injection) for a proliferation marker (Phos-H3) (d) and apoptosis (cleaved Caspase3) (e) (both showed in red). Nuclei are stained with DAPI (blue). 


\section{Extended Data Figure 2}

a

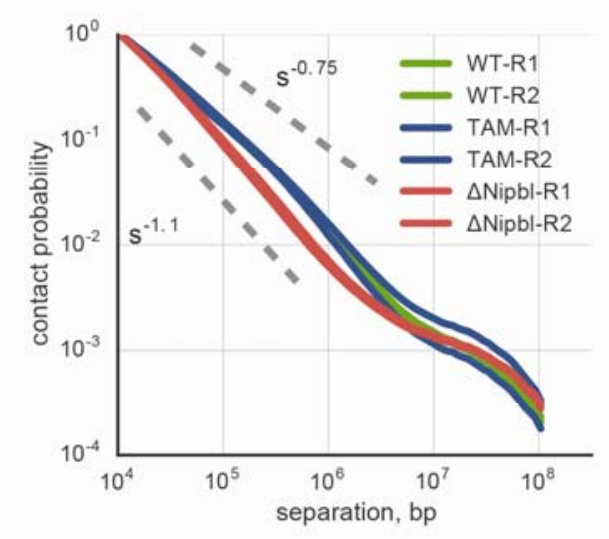

b

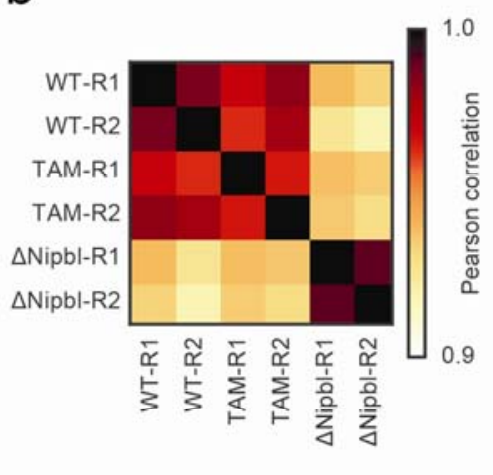

C WT TAM $\Delta \mathrm{Nipbl}$

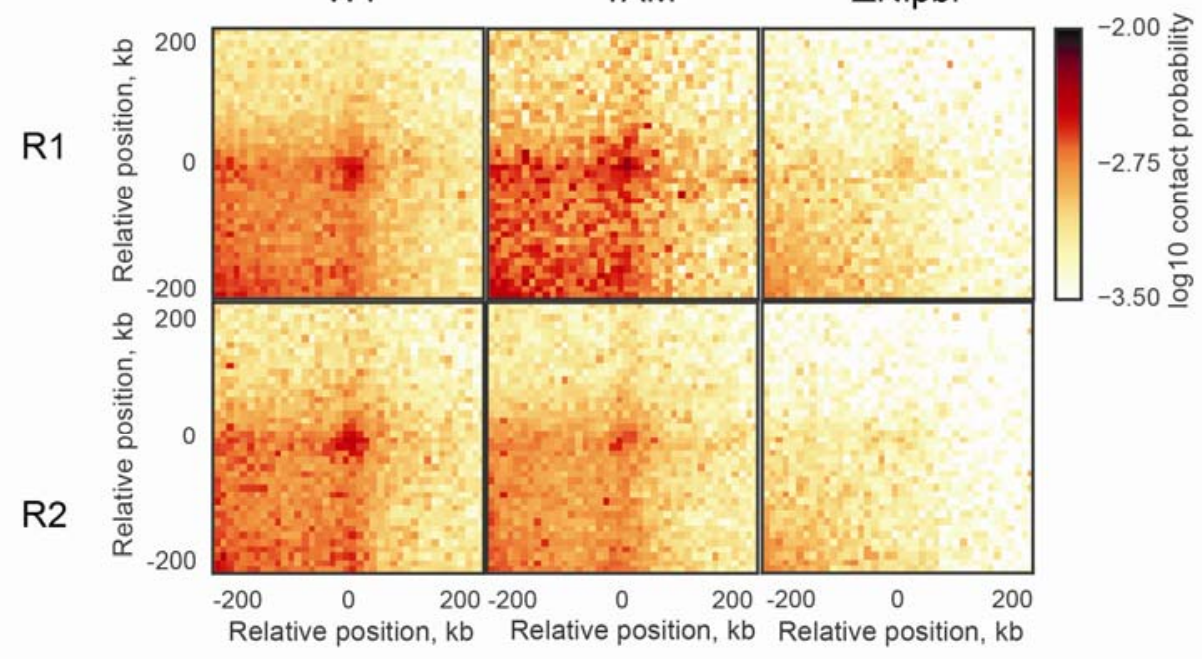

\section{Extended Data Figure 2. The Hi-C maps obtained in all experimental conditions show} extensive similarities between replicates. (a) The scaling curves of contact probability $\mathrm{P}(\mathrm{s})$ vs genomic distance s, normalized to unity at $10 \mathrm{~kb}$ separation. (b) The matrix of Pearson correlation coefficients between cis autosomal eigenvectors of replicates detected at 100kb. (c) The average Hi-C maps of $102500 \mathrm{~kb}-600 \mathrm{~kb}$ loops ${ }^{8}$ in each replicate of each experimental condition. 


\section{Extended Data Figure 3}

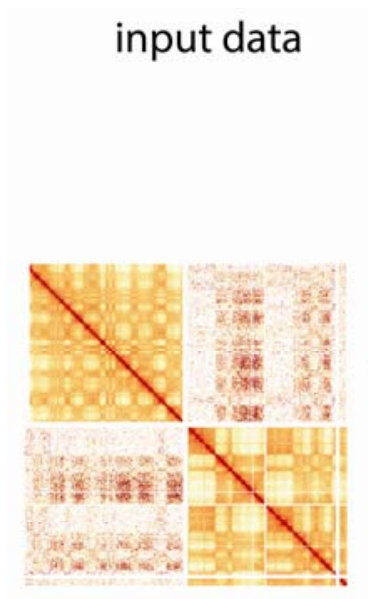

whole intraand inter-chromosomal contact maps

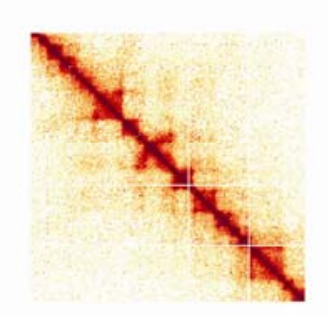

short-distance (<few Mb)

intra-chromosomal contact map feature

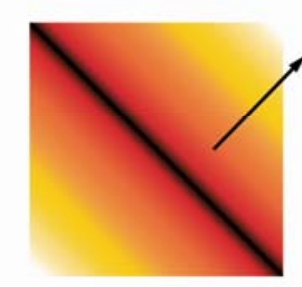

decay of contact frequency with genomic distance

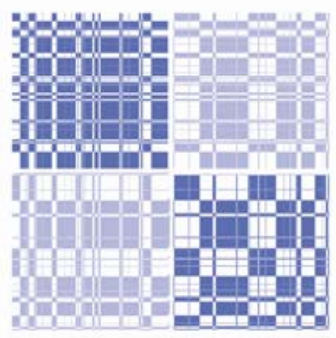

checkered pattern in cis and trans

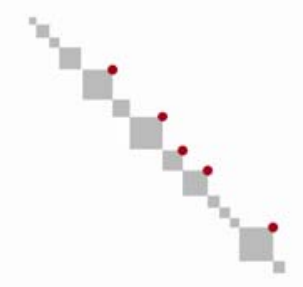

domains of local contact enrichment

\section{quantification}

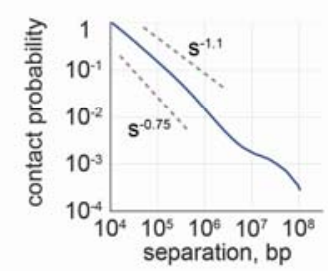

scaling curves
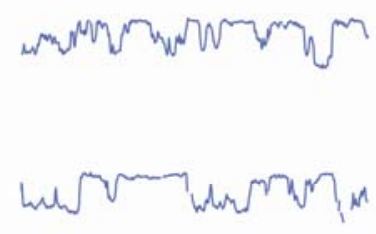

compartment track

list of

TADs, loops

\section{Extended Data Figure 3. Overview of various features of chromosomal architecture} detected and quantified in Hi-C contact maps. Top row - intra-chromosomal maps show the decay of contact frequency with genomic distance, which can be quantified with the curves of contact frequency $\mathrm{P}(\mathrm{s})$ vs genomic separation s. Middle row - both intra- and inter-chromosomal maps display a checkered pattern caused by compartmentalization of the genome. This pattern can be quantified by a continuous genomic track obtained via eigenvector decomposition of either cis or trans maps. Bottom row - intra-chromosomal maps at short genomic distance scales reveal domains of enriched contact frequency, which appear as bright squares along the main diagonal, and loops which appear as bright dots connecting two loci. Both can be detected and quantified using specialized algorithms. 


\section{Extended Data Figure 4}

a
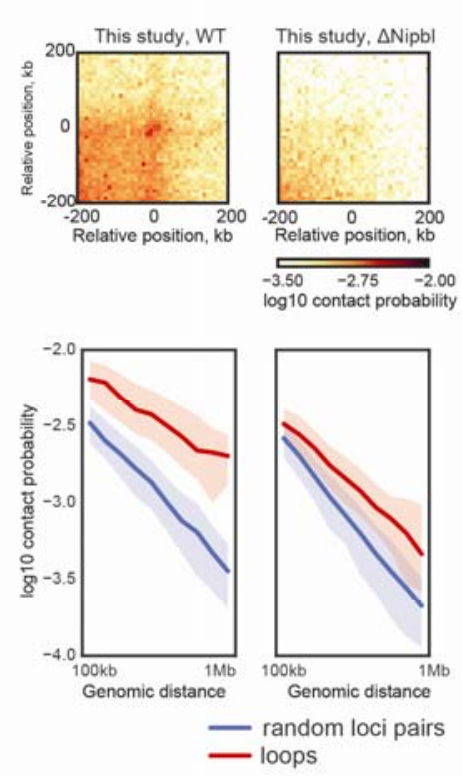

b
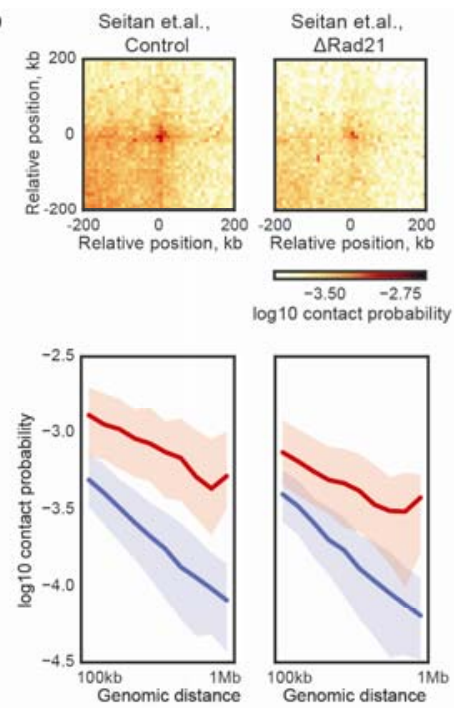

d
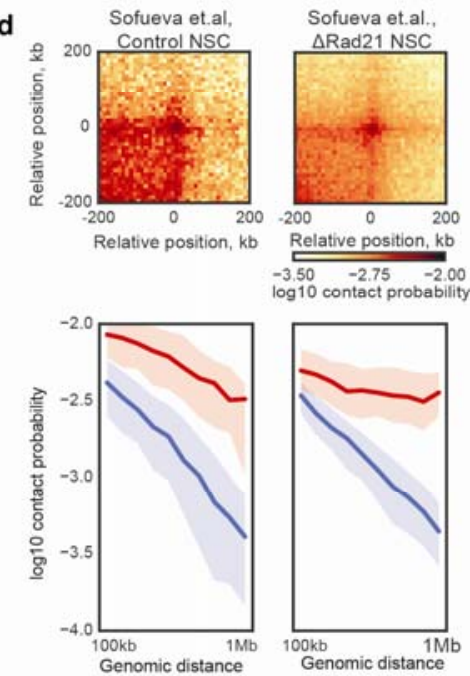

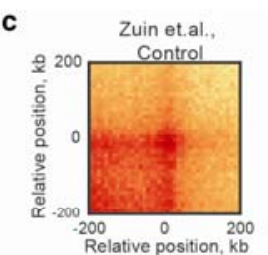

Zuin et.al.
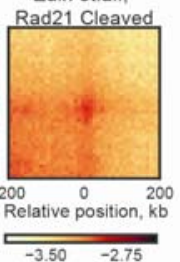

$\log 10$ contact probability
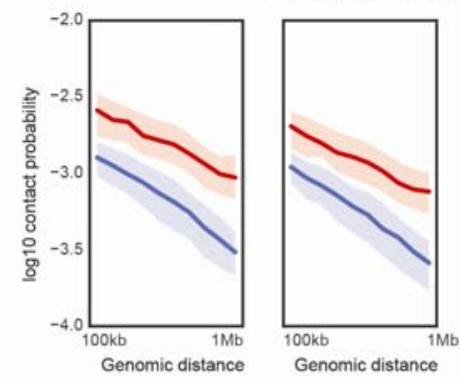

e

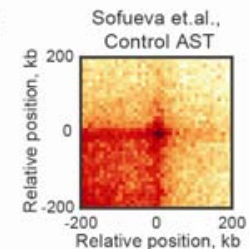

Sofueva et.al,
$\triangle$ Rad21 AST
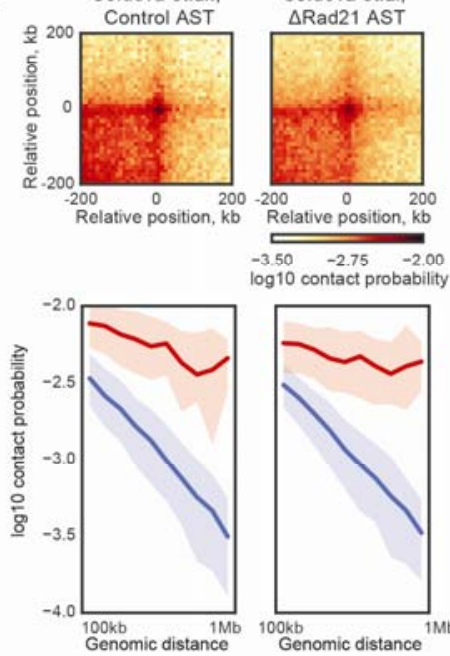

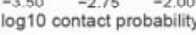

Extended Data Figure 4. Deletion of $\mathrm{Nipbl}$ in this study leads to a robust disappearance of loops compared the techniques previously used to deplete chromatin-bound cohesin. (a) genetic deletion of $\mathrm{Nipbl}$ in hepatocytes, this study. (b) deletion of Rad21 in thymocytes ${ }^{19}$. (c) proteolytic cleavage of RAD21 in HEK293T cells ${ }^{17}$ (d-e) deletion of Rad21 in NSCs and ASTs ${ }^{18}$. For each dataset: left column, top panels - the average Hi-C map of 102 loops with size range $500-600 \mathrm{~kb}{ }^{8}$ in WT and $\Delta \mathrm{Nipbl}$ contact maps; bottom panels - the relative contact probability between pairs of loop anchors vs genomic distance, compared to randomly selected pairs of loci. The thick line shows the median contact probability; the shading shows the envelope between the $25^{\text {th }}$ and $75^{\text {th }}$ percentiles of contact probability at each genomic separation. 


\section{Extended Data Figure 5}
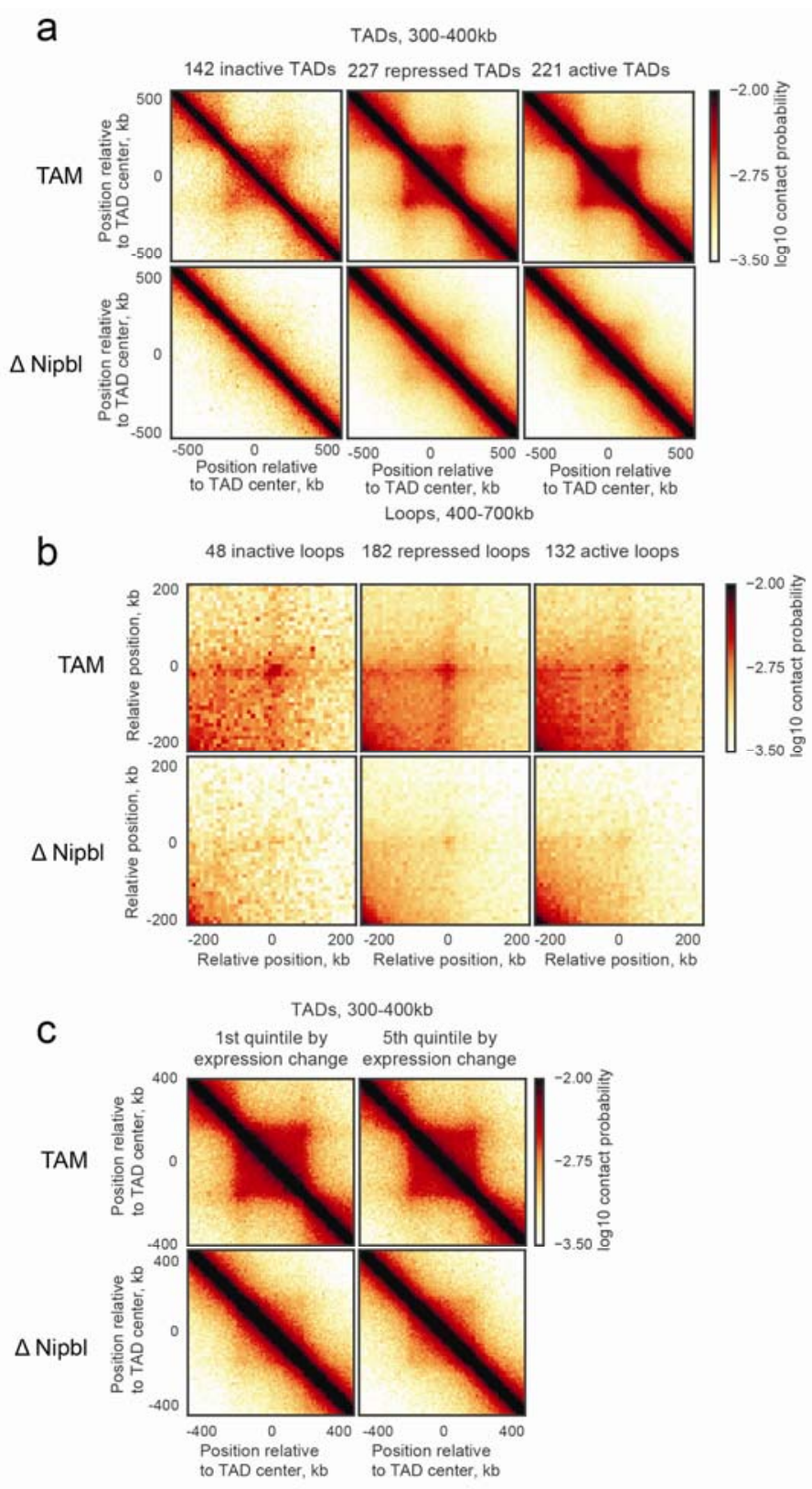

\section{Extended Data Figure 5. Residual structures are observed in active and repressed regions}

of the genome after Nipbl deletion. For each TAD, an activity was assigned based on the dominant simplified ChromHMM state category. (a) The average contact map of 300-400kb TADs in inert, repressed and active regions of the genome. (b) The average contact map of 300$700 \mathrm{~kb}$ loops in inert, repressed and active regions of the genome. (c) The average contact maps of most upregulated 20\% (left) and most downregulated 20\% (right) of 300-400kb TADs. 


\section{Extended Data Figure 6}
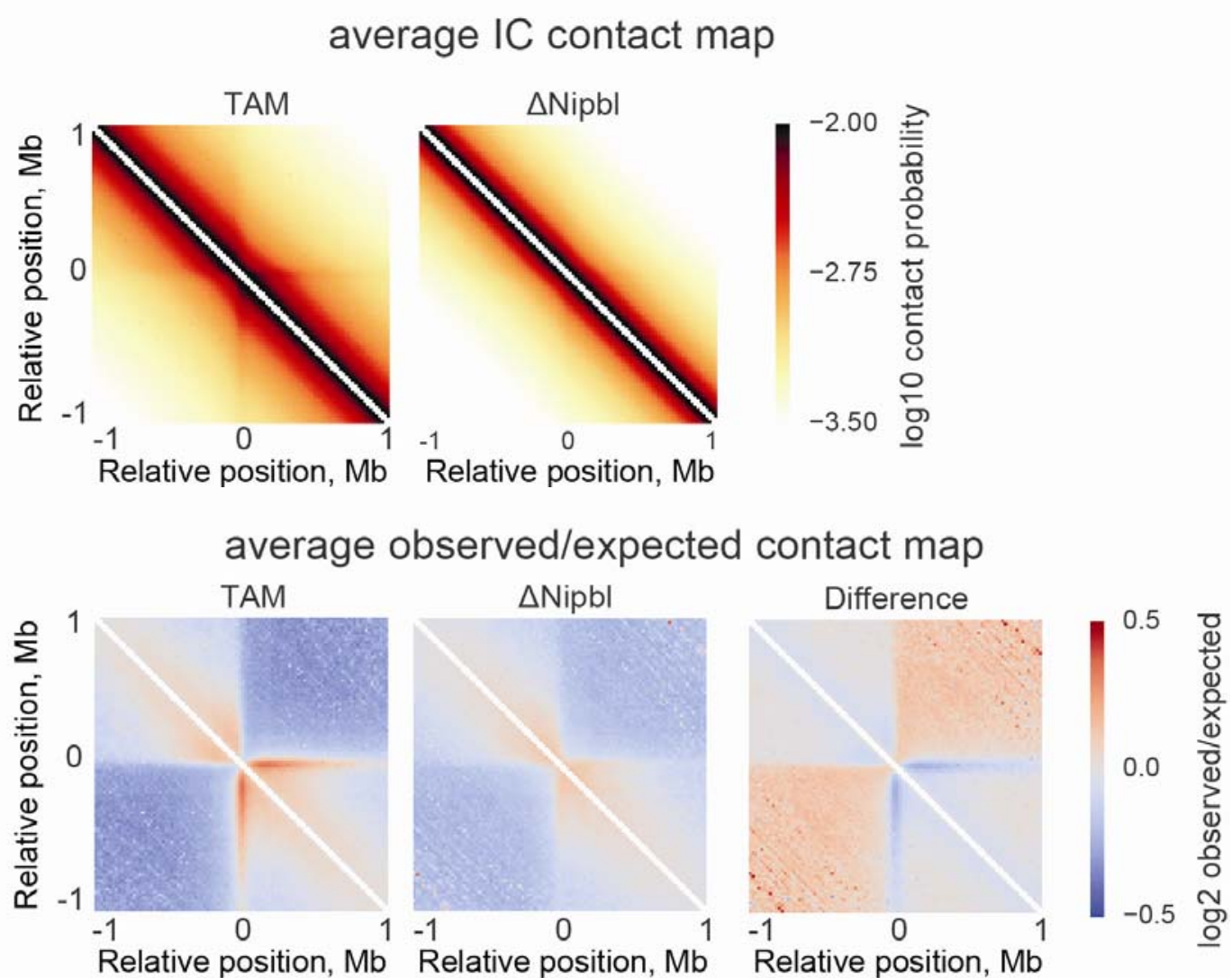

Extended Data Figure 6. The average Hi-C contact map around CTCF peaks. CTCF peaks are taken from ENCODE mouse liver ChIP-Seq data ${ }^{42}$ and supported by an underlying CTCF binding motif occurrence. Top row - average iteratively corrected contact map around 22000 sites in TAM and $\triangle \mathrm{Nipbl}$ cells. Bottom row - average contact map normalized by the expected contact frequency at a given genomic separation. 


\section{Extended Data Figure 7}

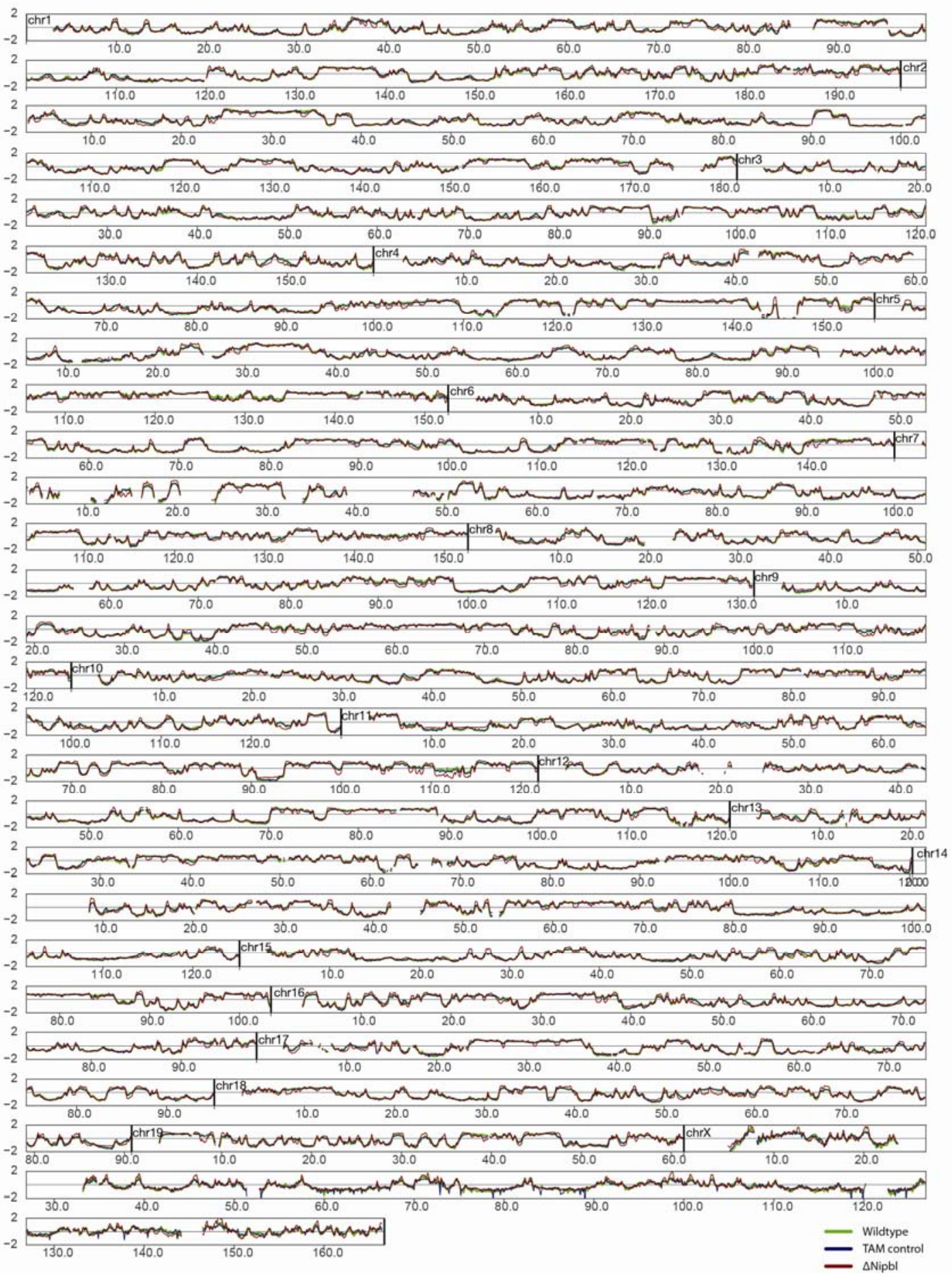

Extended Data Figure 7. The genome-wide eigenvectors calculated from $20 \mathrm{~kb}$ cis contact matrices in all three experimental conditions, WT (green), TAM (blue), $\Delta \mathrm{Nipbl}$ (red). 


\section{Extended Data Figure 8}

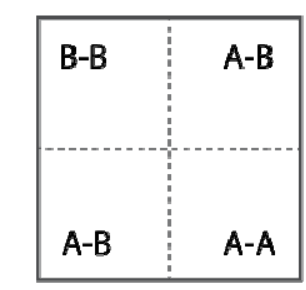

Cis contact frequency

Trans contact frequency
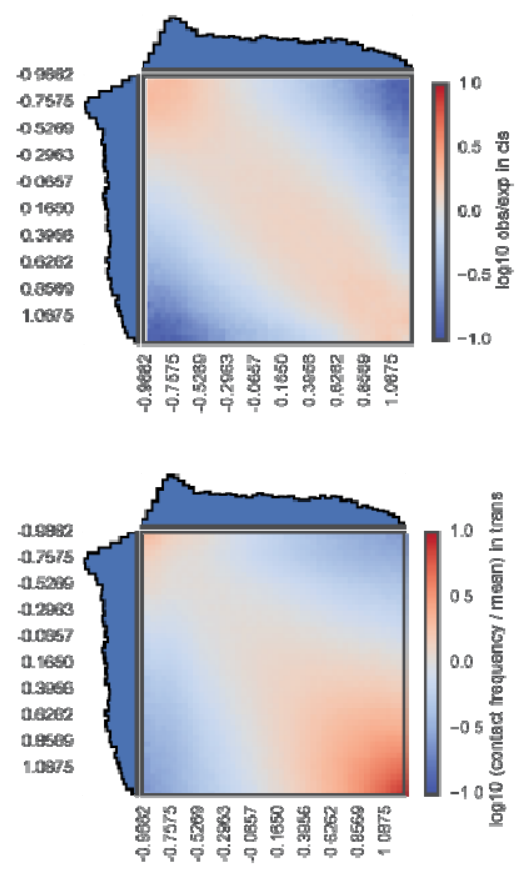

$\Delta \mathrm{Nipbl}$
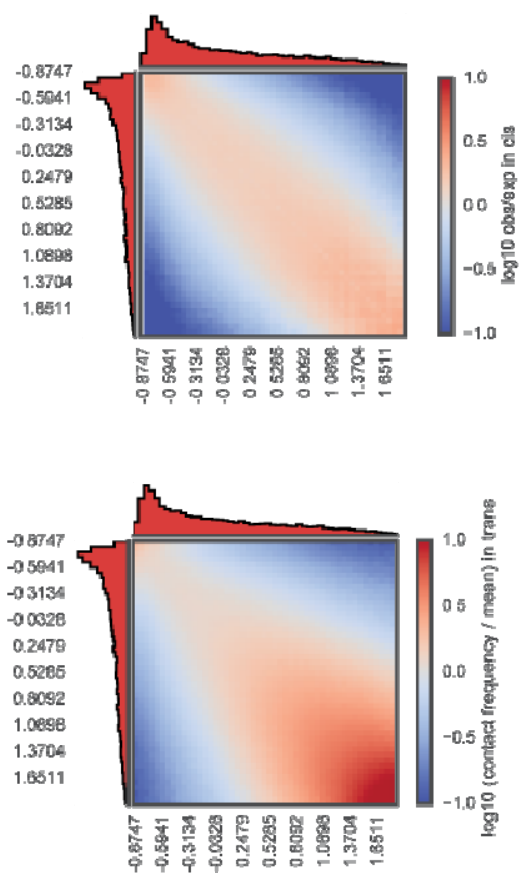

Extended Data Figure 8. Increased compartmentalisation in $\Delta$ Nipbl cells. Average interaction frequencies between pairs of loci (100kb bins) arranged by their compartment signal (eigenvector value). Notice enrichment of $\mathrm{AA}$ and depletion of $\mathrm{AB}$ interactions in $\triangle \mathrm{Nipbl}$ cells: see the diagram for $\mathrm{AA}, \mathrm{AB}, \mathrm{BB}$ regions. The interaction frequencies in cis (top row) are computed for observed/expected contact maps. Histograms along the axes show the distributions of eigenvector values. 


\section{Extended Data Figure 9}

a

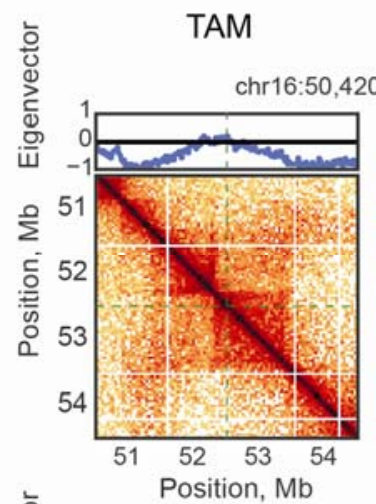

b

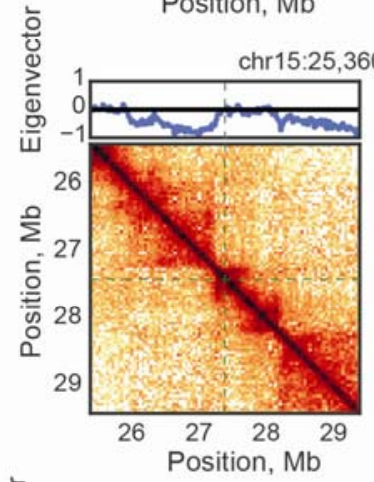

c

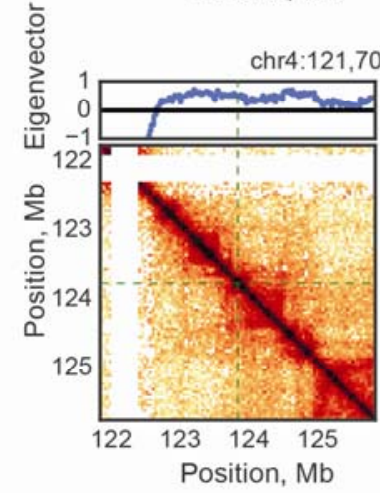

$\Delta \mathrm{Nipbl}$

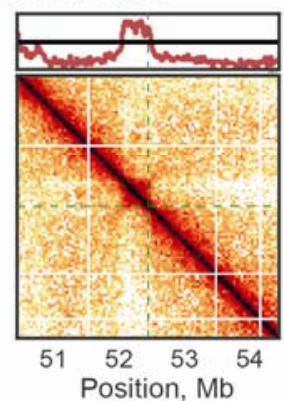

Position, Mb
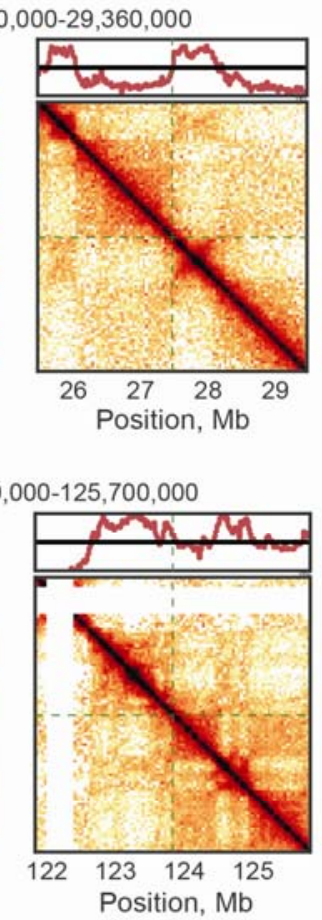

d
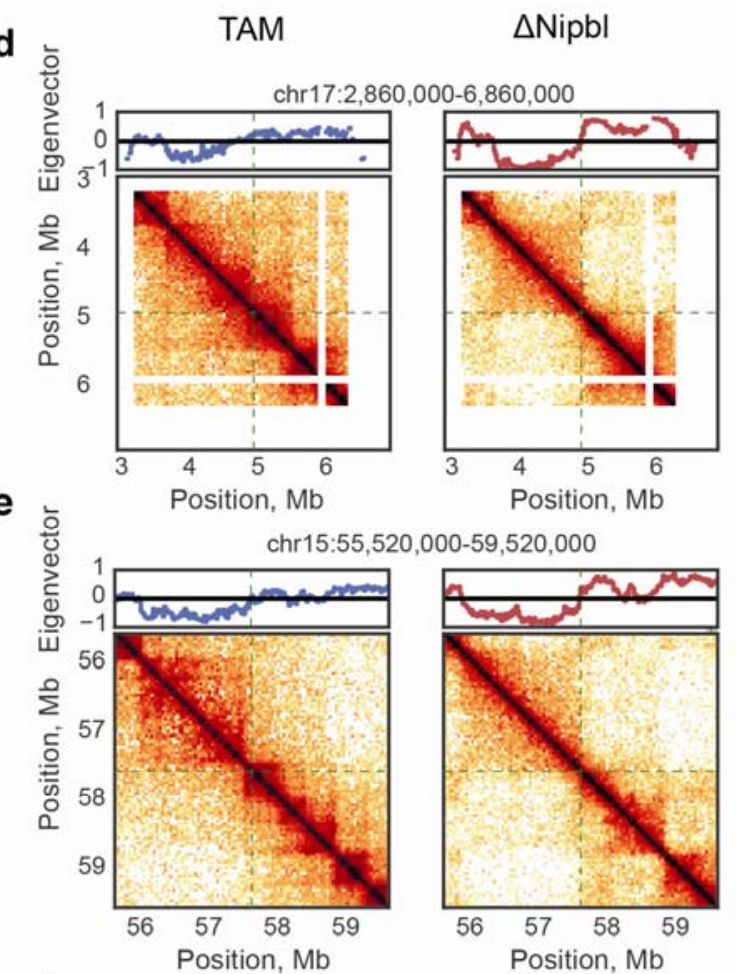

f

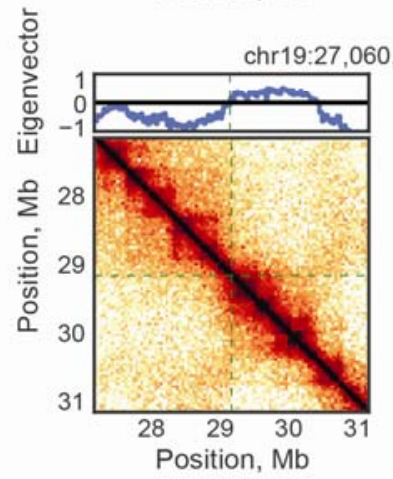

Position, Mb

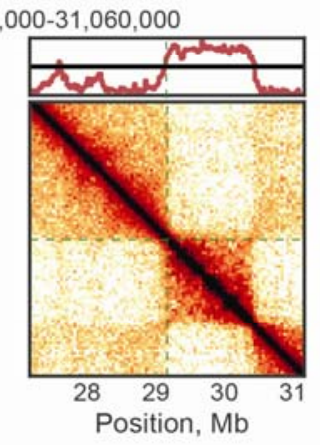

Extended Data Figure 9. Boundaries of former TADs and new compartments do not coincide. Examples of TADs detected in WT cells which cross sharp compartment transitions revealed by $\triangle \mathrm{Nipbl}$ contact maps. Left column - TAM control data, right column - $\Delta$ Nipbl data. Top of each figure - local eigenvector track in the corresponding cell type. The contact maps are centered at the sharp compartment transition in $\Delta$ Nipbl. These examples illustrate that that chromatin-bound cohesins can locally interfere with genome compartmentalization. 


\section{Extended Data Figure 10}
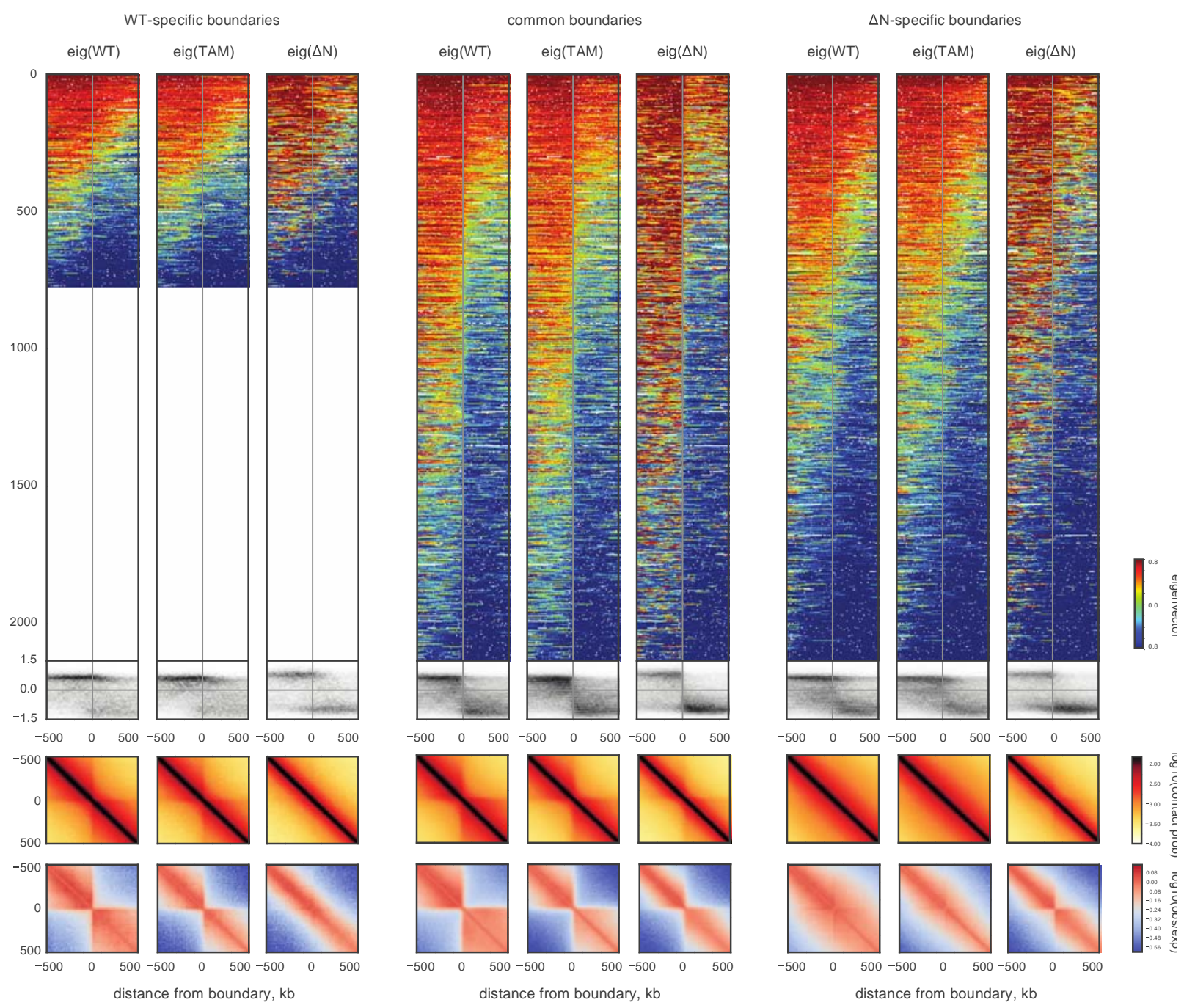

\section{Extended Data Figure 10. The residual contact-insulating boundaries in $\Delta$ Nipbl are} associated with compartment transitions. The first group of columns considers the boundaries detected in WT cells only, the second pair considers boundaries detected both in WT and $\Delta \mathrm{Nipbl}$ cells, the last pair considers boundaries detected in $\Delta \mathrm{Nipbl}$ only. For each group, the first, second and third columns display data (eigenvectors and Hi-C) from WT, TAM and $\triangle$ Nipbl cells, respectively. Within each column: the top row - a stack of eigenvector tracks in a +/- 500kb window around boundaries, oriented such that the left-half of the window has greater average signal value and sorted by the average WT eigenvector value in the window. The second row density histogram of eigenvector values as a function of the distance to the boundary. The third 
and fourth row - the boundary-centered average contact probability and observed/expected contact ratio, respectively. The density histograms show that common and $\Delta$ Nipbl-specific boundaries correspond to sharp transitions of compartment signals in $\Delta \mathrm{Nipbl}$ cells, in contrast to the more diffuse signal at these positions in WT and TAM cells. 
bioRxiv preprint doi: https://doi.org/10.1101/094185; this version posted December 15,2016 . The copyright holder for this preprint (which was not certified by peer review) is the author/funder, who has granted bioRxiv a license to display the preprint in perpetuity. It is made available under aCC-BY 4.0 International license.

\section{Extended Data Figure 11}

a
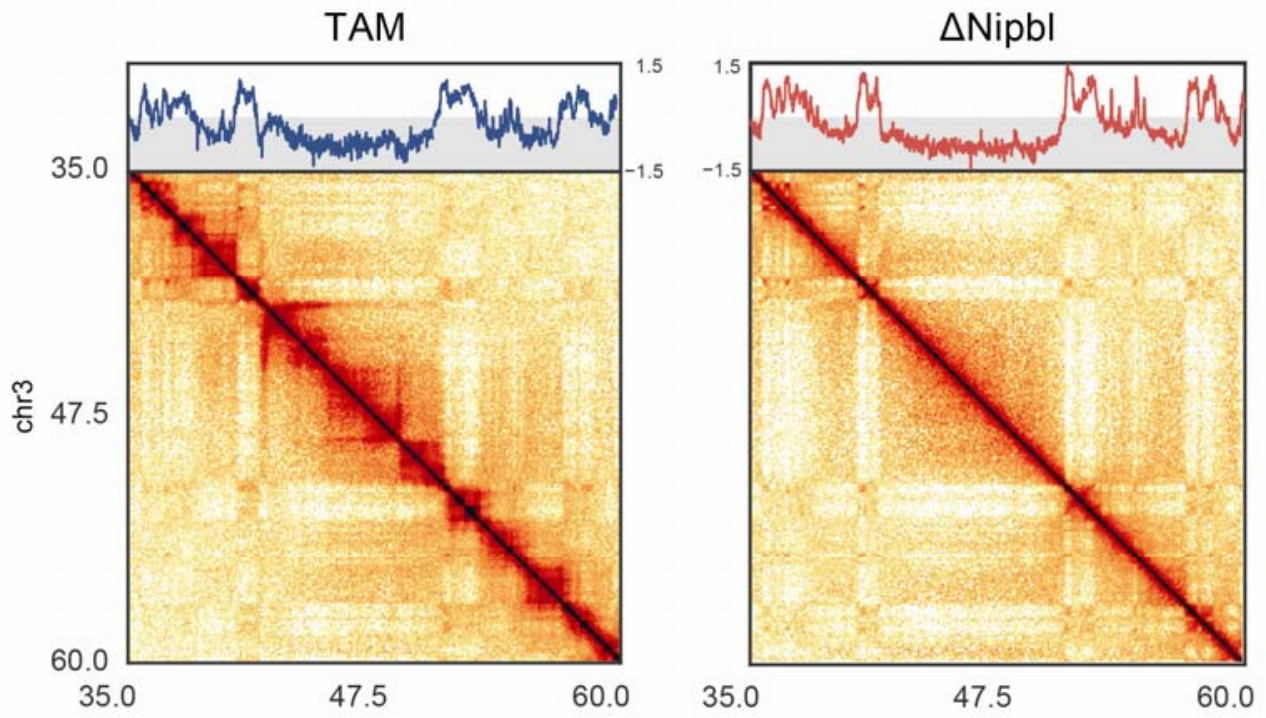

b
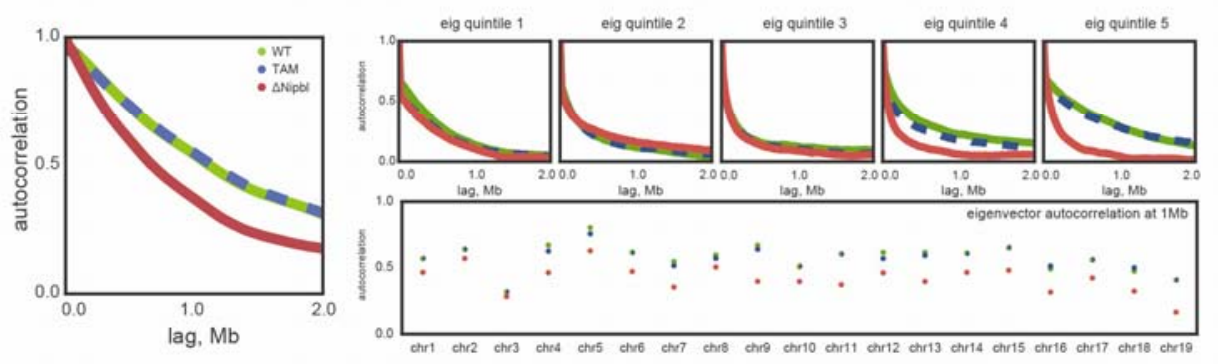

C
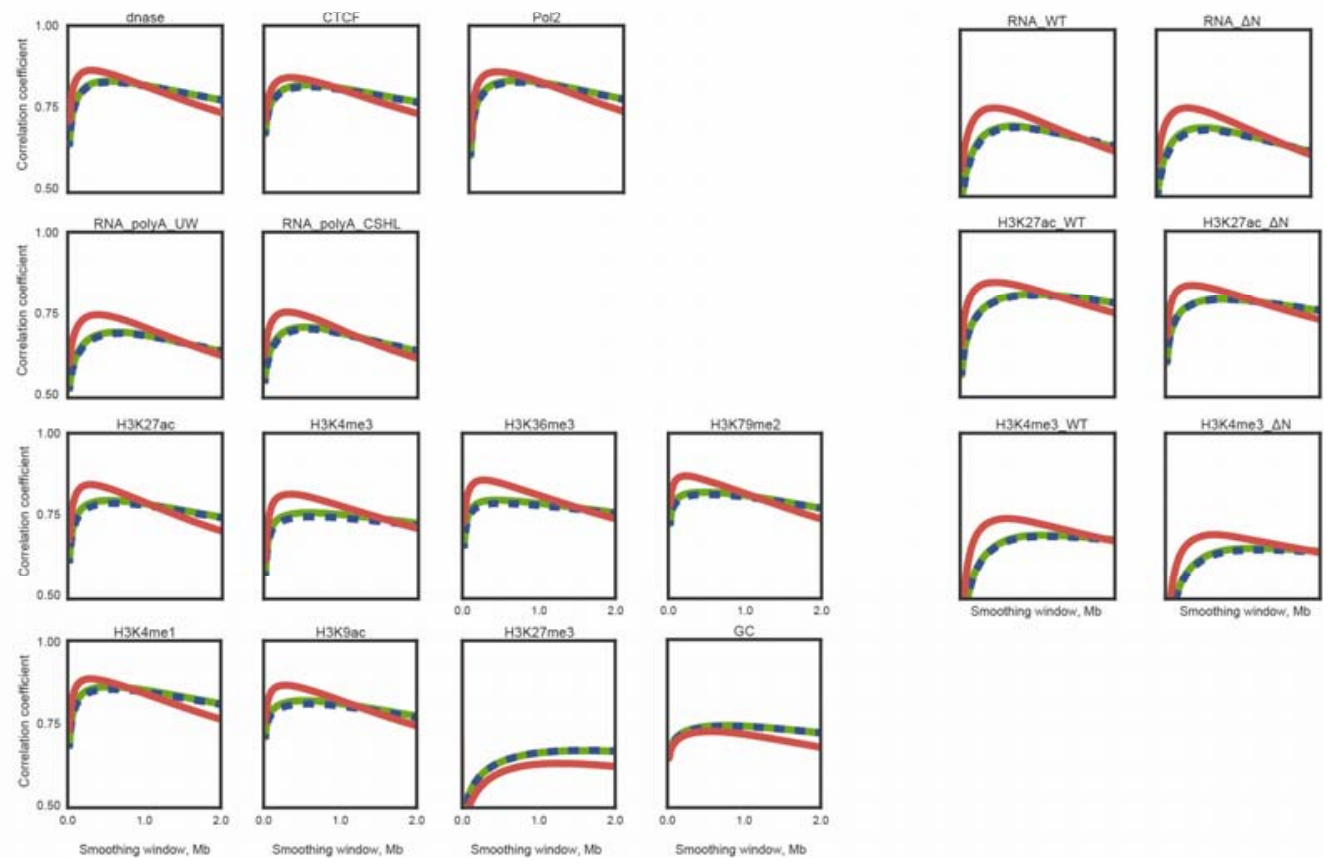
Extended Data Figure 11. Fragmentation upon Nipbl deletion in smaller alternating regions of $\mathbf{A}$ and $\mathbf{B}$ compartment-type is activity-dependent. (a) Example region (chr3:35-60Mb) illustrating lack of compartment fragmentation in uniformly B-rich regions yet robust disappearance of TADs. Top - compartment eigenvector, Bottom - contact matrix snapshot. (b) Autocorrelation of eigenvector tracks reveals genome-wide fragmentation of active compartments. Left - the genome-wide correlation of the $20 \mathrm{~kb}$ cis eigenvector values of pairs of loci as a function of their genomic separation (autocorrelation). Top right - eigenvector correlation of locus pairs split by quintile of the eigenvector value of the upstream locus. Bottom right - chromosome-wide values of eigenvector correlation of locus pairs separated by $1 \mathrm{Mb}$. (c) Correlation between the smoothed histone and TF ChIP-seq and RNA-seq tracks and the 20kb cis eigenvectors as a function of the smoothing window size. Left group of panels - ENCODE data, right - data from this study. First and second rows - histone marks, third row - RNA-seq tracks, fourth row - miscellaneous tracks (DNase hypersensitivity, CTCF and PolII ChIP-seq and GC content). $\triangle \mathrm{Nipbl}$ eigenvectors show an increased correlation with tracks associated with transcriptional activity yet a decreased correlation with the repression-associated track of H3K27me3 and GC content. 


\section{Extended Data Figure 12}

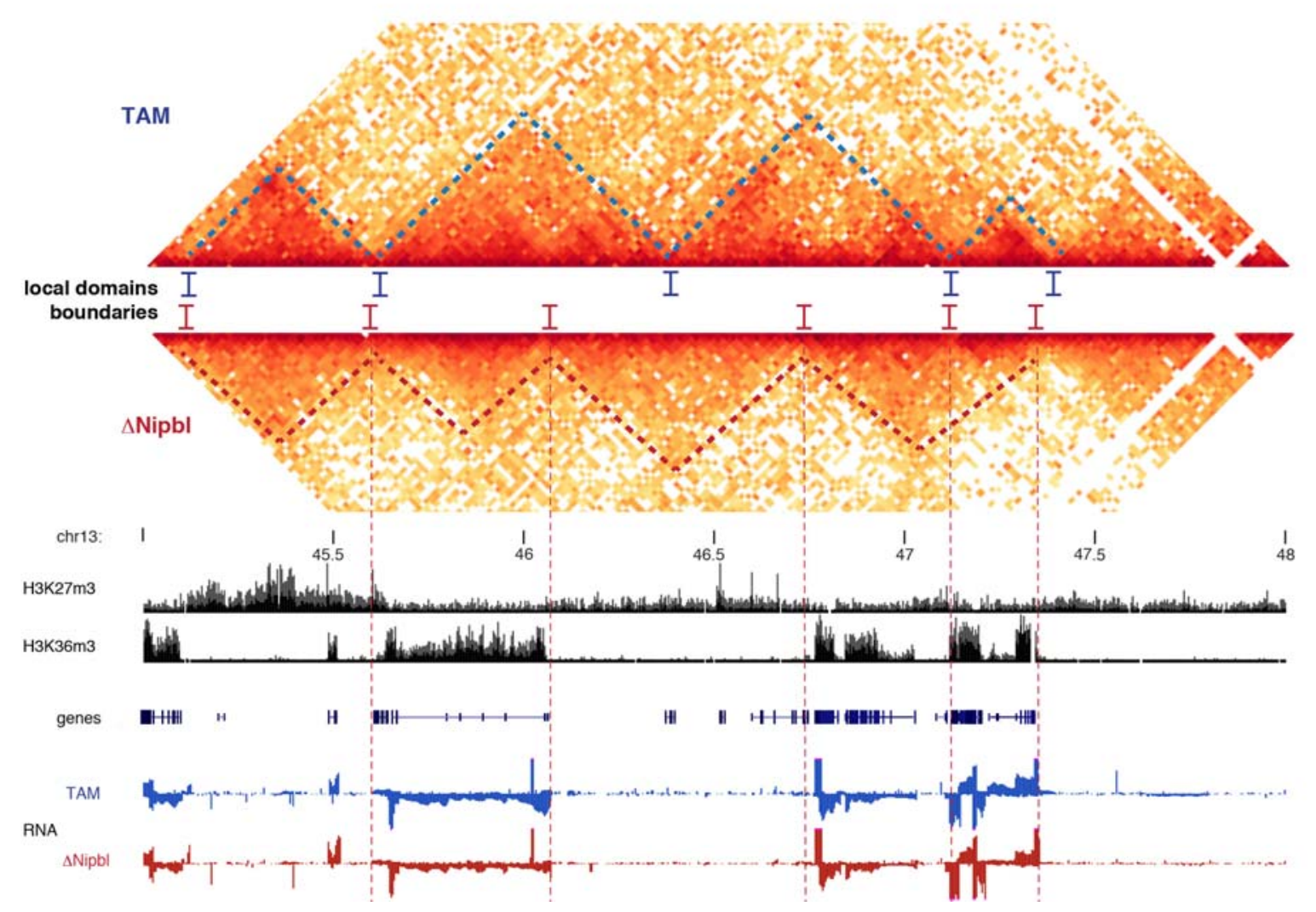

Extended Data Figure 12. New structures appear in $\Delta$ Nipbl cells and follow the underlying chromatin activity. Example of a large WT A-type compartment region (chr13:45-48Mb). Hi-C maps show different structures (TADs) highlighted by dashed lines (upper panel, TAM control, blue lines; lower panel, $\Delta \mathrm{Nipbl}$, red line). Boundaries are shifted or lost and replaced by new ones in $\Delta \mathrm{Nipbl}$ cells. Histone ChIP-seq tracks ${ }^{42}$ and stranded RNA-seq tracks (blue: TAM hepatocytes, red; $\triangle \mathrm{Nipbl}$ cells) highlight that WT/TAM TADs do not strictly follow the underlying chromatin activities, whereas the new structures in $\Delta \mathrm{Nipbl}$ cells delineated by red dashed lines correspond precisely to active versus inactive chromatin domains. 


\section{Extended Data Figure 13}
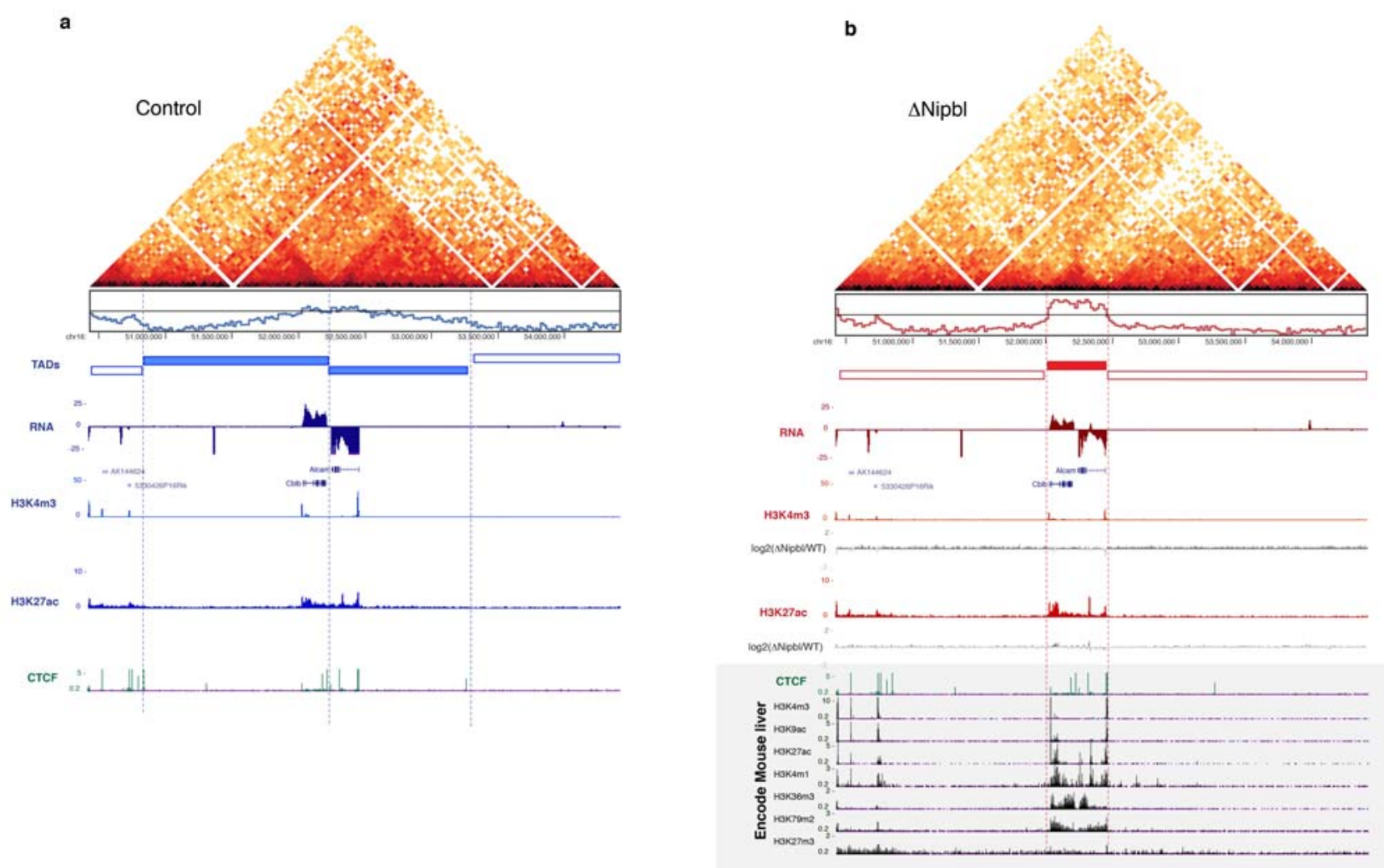

\section{Extended Data Figure 13. New compartments do not respect TAD boundaries but the}

underlying chromatin domains. A large region (chr16:50420000-54420000) adopts a very different $3 \mathrm{D}$ organisation in control (a) (in blue) and $\Delta \mathrm{Nipbl}$ cells (b) (in red). Hi-C data are shown, as well as the eigenvector values in the two conditions. RNA-Seq tracks showed minimal changes of expression (Alcam expression is reduced by 2 -fold in $\Delta \mathrm{Nipbl}$ cells) and chromatin states. ChIP-Seq tracks for H3K27ac and H3K4me3 are shown in the two conditions, with $\log 2$ ratio tracks under the $\Delta \mathrm{Nipbl}$ (b) panel. Encode tracks (corresponding to WT liver cells) are shown in the grey boxed area. The new structure adopted in $\Delta$ Nipbl cells put together the two active genes which are normally in different TADs in the same domain, corresponding to the active chromatin linear domain. 


\section{Extended Data Figure 14}

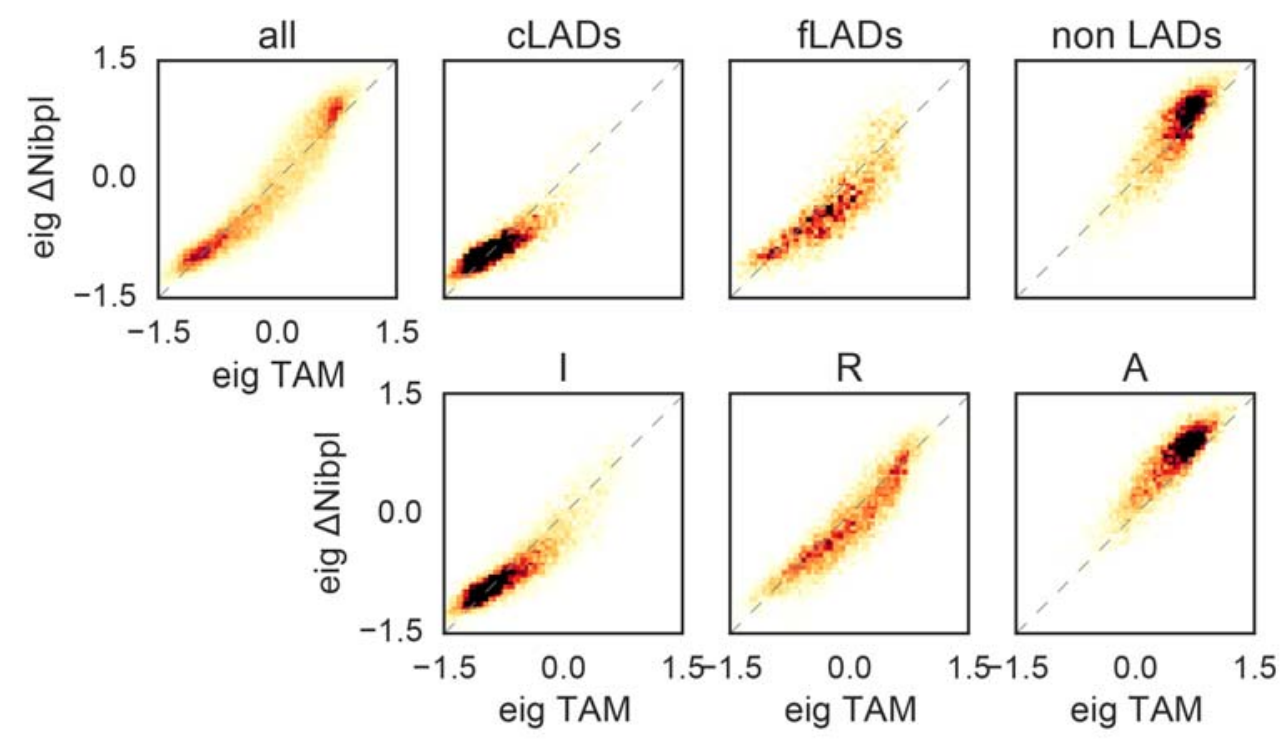

Extended Data Figure 14. Correlation of cis eigenvector values of $100 \mathrm{~kb}$ genomic bins before and after Nipbl deletion, split by the functional state of chromatin. Top row, left to right: genome-wide relationship; bins showing constitutive lamin-B1 association across 4 mouse cell types (cLADs); bins showing variable (facultative) lamin-B1 association (fLADs); binds not showing any association (non LADs). Bottom row: bins assigned the Inert ChromHMM simplified state; bins assigned the Repressed state; bins assigned the Active state. 


\section{Extended Data Figure 15}

a

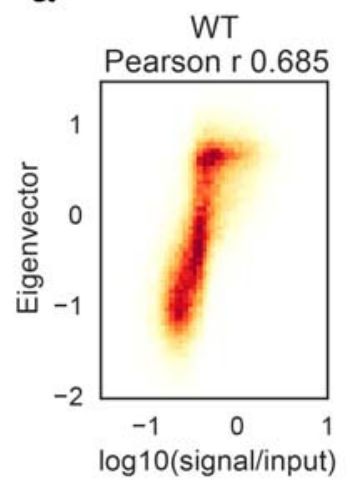

H3K27ac ChIP-Seq

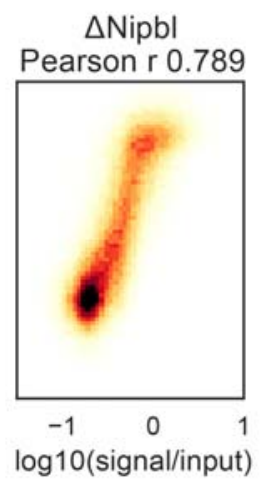

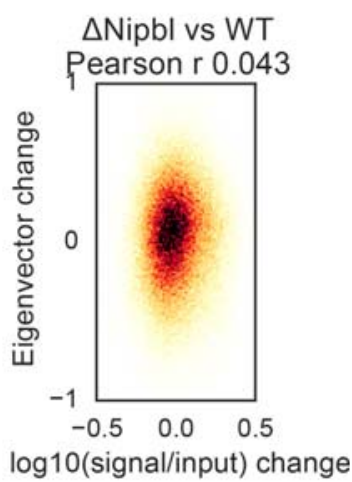

b $\begin{gathered}\text { RNA-seq expression RPM } \\ \triangle \text { Nipbl - TAM }\end{gathered}$

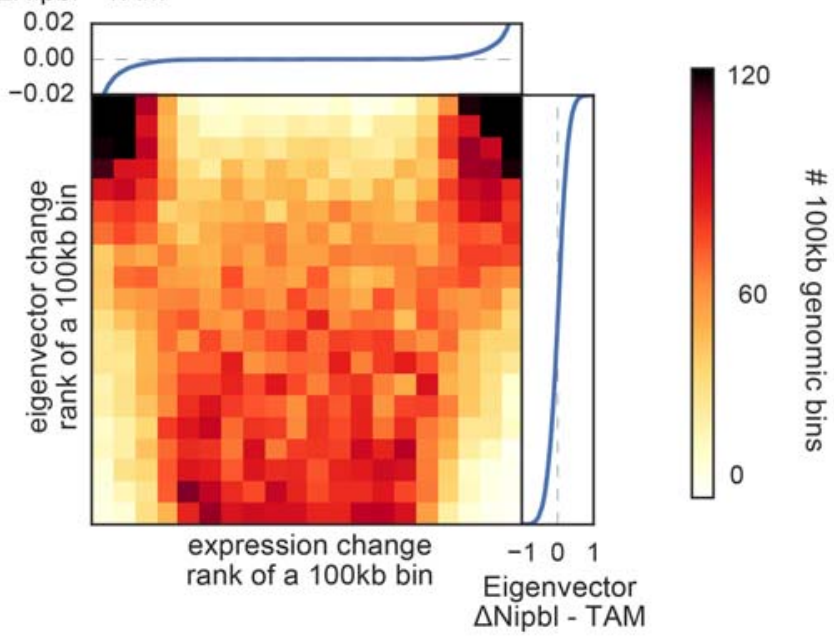

Extended Data Figure 15. Eigenvector change upon Nipbl deletion is uncorrelated with changes in gene expression or epigenetic marks. (a) ChIP-Seq signal for histone marks of activity vs eigenvector value of $20 \mathrm{~kb}$ bins, top row - H3K27ac, bottom row - H3K4me3. Left column - WT cells, middle column - $\Delta \mathrm{Nipbl}$ cells, right column - correlation of changes in both signals upon Nipbl deletion. (b) The change in the compartment structure upon Nipbl deletion cannot be attributed to the sign of the local expression change. The heatmap shows the number of $100 \mathrm{~kb}$ genomic bins as a function of the ranks of expression change and the eigenvector change. The attached plots show the correspondence between the values of expression change (top) or eigenvector change (right) and their ranks. 
bioRxiv preprint doi: https://doi.org/10.1101/094185; this version posted December 15,2016 . The copyright holder for this preprint (which was not certified by peer review) is the author/funder, who has granted bioRxiv a license to display the preprint in perpetuity. It is made available under aCC-BY 4.0 International license.

\section{Extended Data Figure 16}

a

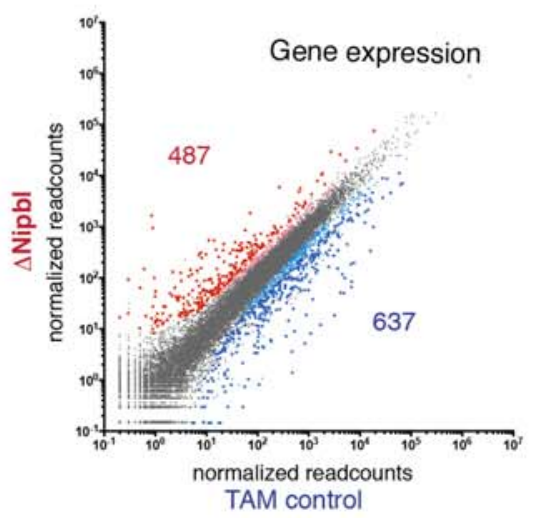

C

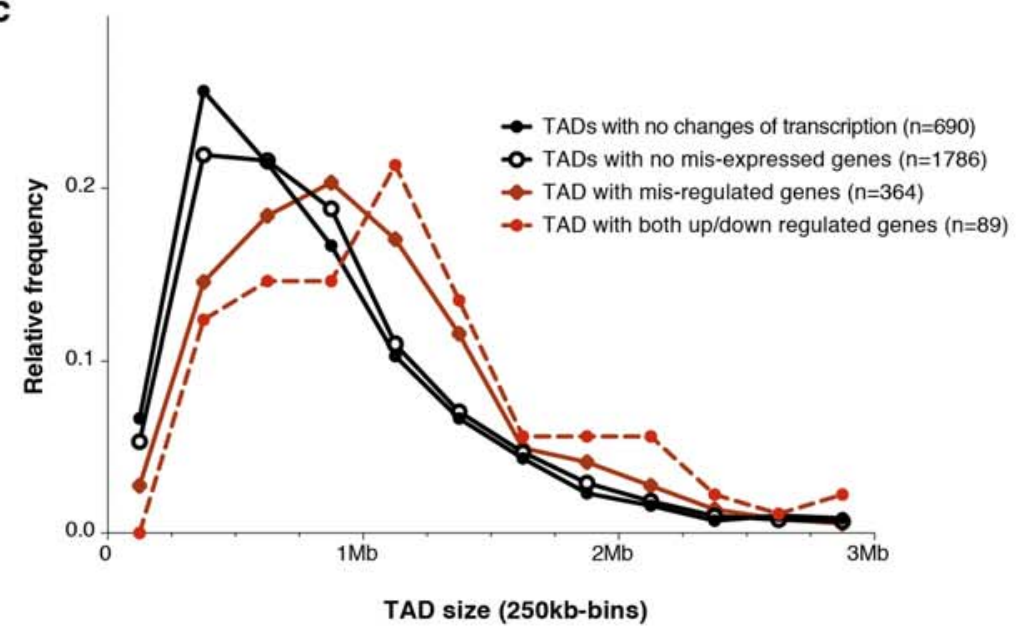

b

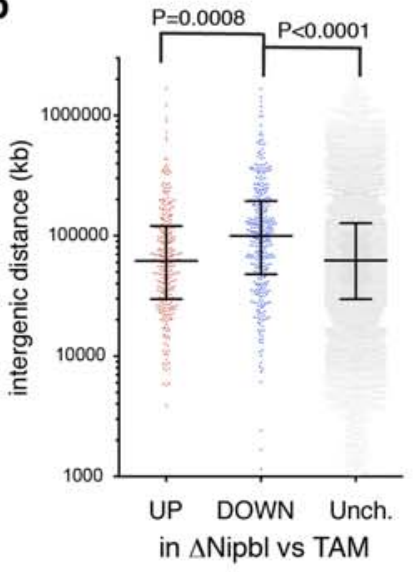

d

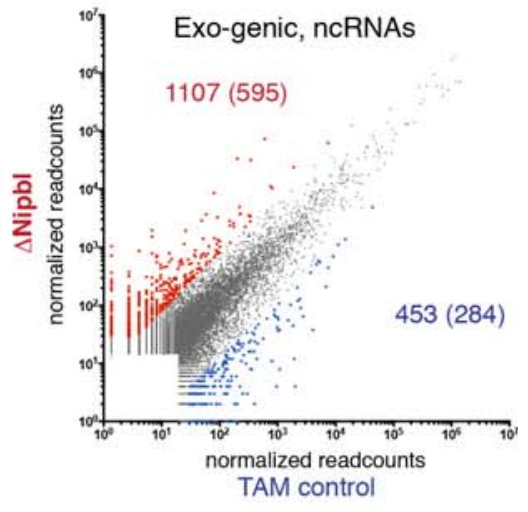

e

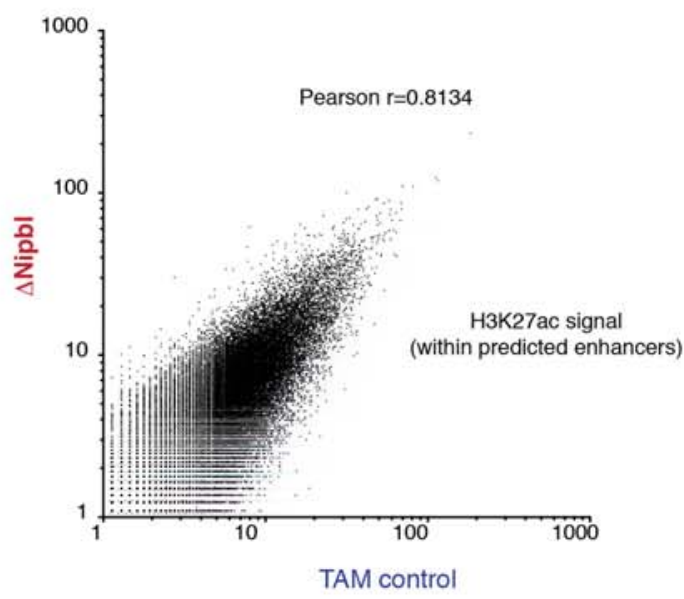


Extended Data Figure 16 - Expression changes in $\Delta$ Nipbl hepatocytes. (a) Changes in gene expression between TAM controls and $\triangle \mathrm{Nipbl}$ liver cells (four replicates for each condition). Genes with significant changes in gene expression (FDR > 0.05) are coloured in red (upregulated) or blue (down-regulated), with larger dots corresponding to gene with a fold-change > 3 (numbers given correspond to these high-confidence subset of dis-regulated genes). (b) Intergenic distances for the different categories of dysregulated genes. Statistical differences determined by an unpaired two-tailed $t$-test. (c) Size distribution of the TADs observed in WT (lost in $\Delta \mathrm{Nipbl}$ ) depending on the degree alteration of their transcriptional states. The size of TAD with transcriptional changes (red) is significantly larger than those that do not show transcriptional alterations (black) (Kolmogorov-Smirnov, $P<0.0001)$ (d) Change in transcription in non-genic intervals (including inter-genic and antisense within gene bodies). Gene expression was calculated as the normalized number of read within intervals defined by merging adjacent $1 \mathrm{~kb}$ windows showing readcounts over background (see Methods). The numbers of non-coding transcription up-regulated (in red) or down-regulated (in blue) in $\triangle \mathrm{Nipbl}$ compared to the TAM control is given ( $P$-value $<0.01$, fold-change higher than 8$)$, with the second number indicating the high-confidence events (labelled with coloured dots, expression value over an arbitrary threshold of 30 reads) which constitute the list used for subsequent analyses. (e) Comparison of control and $\triangle \mathrm{Nipbl} \mathrm{H} 3 \mathrm{~K} 27 \mathrm{ac}$ normalized signals within predicted liver enhancer elements (readcounts within $+/-500$ bp of predicted enhancer peak $)^{42}$. 


\section{Extended Data Figure 17}

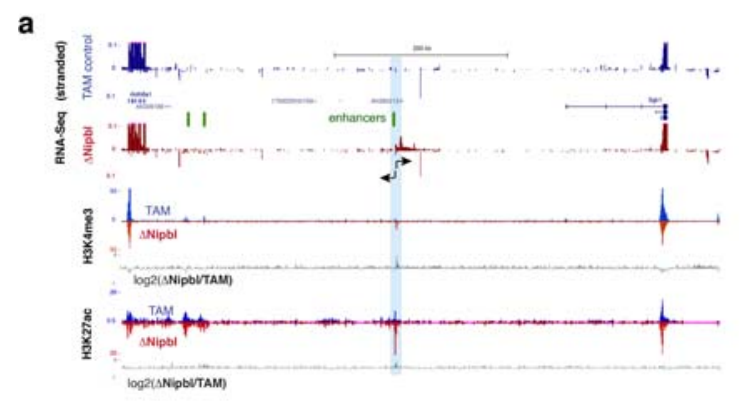

b

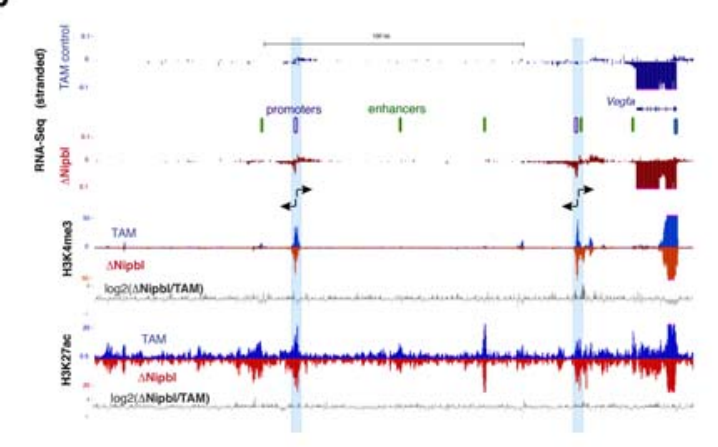

c

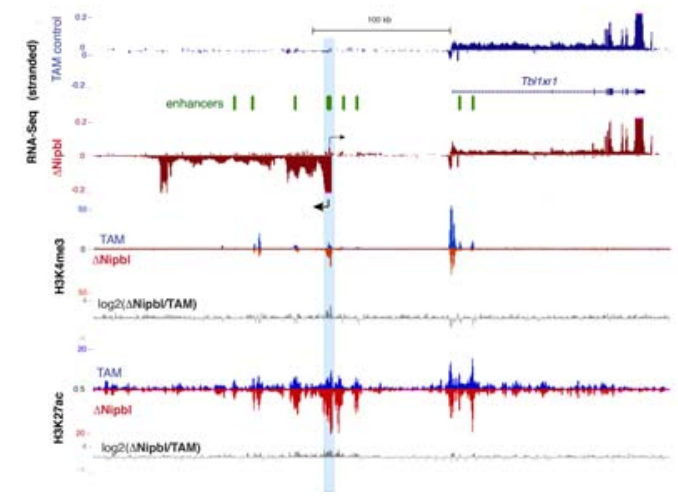

d

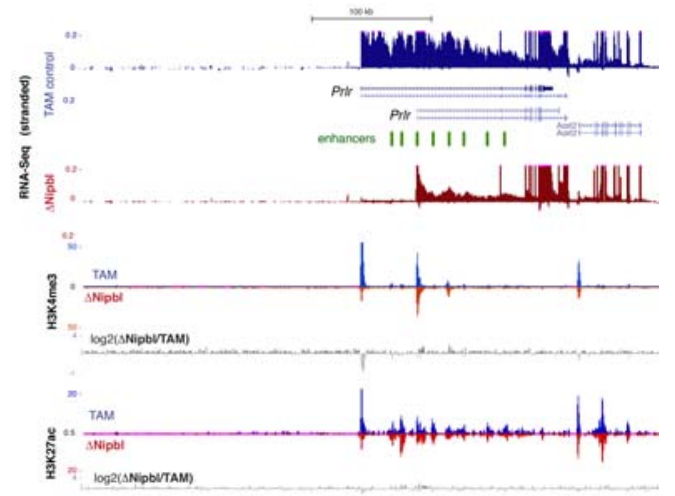

e
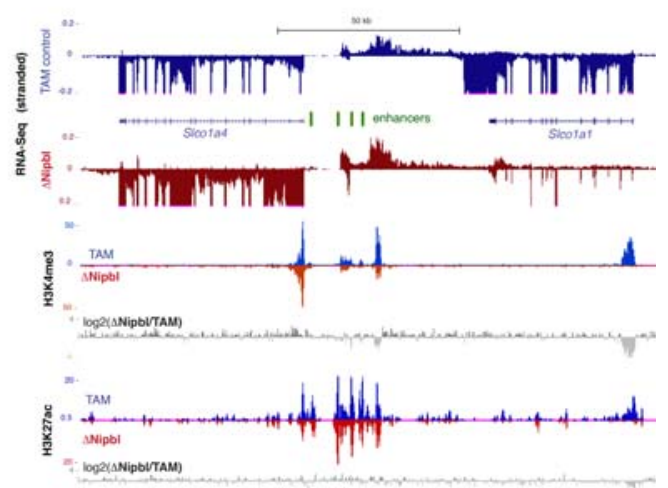

Extended Data Figure 17. Transcriptional changes upon Nipbl deletion. Stranded RNA-Seq and ChIP-Seq tracks (H3K4me3, H3K27ac) are shown for control (blue) and $\Delta$ Nipbl (red) samples. Comparison of the chromatin profiles are shown with $\log 2(\Delta \mathrm{Nipbl} / \mathrm{TAM})$ tracks for H3K4me3 and H3K27ac (in grey). Active enhancers (peaks of high H3K27ac, H3K4me1 ${ }^{42}$, low H3K4me3) are depicted as green ovals. (a) chr10:21,090,000-21,781,000. Bidirectional transcription (position labeled with a blue bar) arises from an isolated enhancer in $\Delta \mathrm{Nipbl}$ cells. (b) chr17:45,945,000-46,176000. Bidirectional transcription (position labeled with a blue bar) arises from two cryptic promoters (H3K4me3 peaks, no/weak transcription in TAM) downstream of Vegfa. (c) chr3:21,712,500-22,126,240. A new transcript from a cryptic promoter $100 \mathrm{~kb}$ 
upstream of Tbllxrl. H3K27ac signal is enhanced at peaks surrounding the activated cryptic promoter. (d) chr15:9,873,000-10,354,700. Promoter switch for Prlr, from an upstream promoter to a more downstream one surrounded by active enhancers. (e) chr6:141,743,961-141,904,692. Downregulation of Slcolal and concomitant up-regulation of Slcola4 and non-coding intergenic transcripts (arrowheads). Distance of Slcola4 promoter to intergenic enhancers is less than 10kb, compared to $80 \mathrm{~kb}$ for Slcolal. 
bioRxiv preprint doi: https://doi.org/10.1101/094185; this version posted December 15,2016 . The copyright holder for this preprint (which was not certified by peer review) is the author/funder, who has granted bioRxiv a license to display the preprint in perpetuity. It is made available under aCC-BY 4.0 International license.

Extended Data Table 1 - TCC libraries

\begin{tabular}{|c|c|c|c|c|}
\hline Replicate & codename & Raw read pairs & Mapped read pairs & Filtered read pairs \\
\hline WT-R1 & 014637Untr & 144640264 & 75465383 & 51745705 \\
\hline WT-R2 & 019440Untr & 117090104 & 60638254 & 43097410 \\
\hline TAM-R1 & 014199TAMcontr & 99057987 & 53285208 & 14530511 \\
\hline TAM-R2 & 014399TAMcontr & 139426858 & 72789581 & 51281408 \\
\hline$\Delta$ Nipbl-R1 & 014200Nipbl & 134157257 & 66635374 & 42405784 \\
\hline$\Delta$ Nipbl-R2 & 016350Nipbl & 139554976 & 69894091 & 50527104 \\
\hline
\end{tabular}


bioRxiv preprint doi: https://doi.org/10.1101/094185; this version posted December 15, 2016. The copyright holder for this preprint (which was not certified by peer review) is the author/funder, who has granted bioRxiv a license to display the preprint in perpetuity. It is made available under aCC-BY 4.0 International license.

\section{Extended Data Table 2 - Public data sources}

\begin{tabular}{|c|c|c|c|c|}
\hline Data type & Description & Cell-type & Ref. & URL \\
\hline \multirow[t]{3}{*}{ Hi-C data } & $\mathrm{Hi}-\mathrm{C}$ raw reads & $\begin{array}{l}\text { Mouse ESC, } \\
\text { NSC, AST }\end{array}$ & 18 & $\begin{array}{l}\text { https://www.ncbi.nlm.nih.gov/geo/query/acc.cgi? } \\
\text { acc=GSE49018 }\end{array}$ \\
\hline & $\mathrm{Hi}-\mathrm{C}$ raw reads & $\begin{array}{l}\text { Mouse } \\
\text { Developing } \\
\text { Thymocytes }\end{array}$ & 19 & $\begin{array}{l}\text { https://www.ncbi.nlm.nih.gov/geo/querv/acc.cgi? } \\
\text { acc=GSE48763 }\end{array}$ \\
\hline & $\mathrm{Hi}-\mathrm{C}$ raw reads & $\begin{array}{l}\text { Human } \\
\text { HEK293T }\end{array}$ & 17 & $\begin{array}{l}\text { https://www.ncbi.nlm.nih.gov/geo/query/acc.cgi? } \\
\text { acc=GSE44267 }\end{array}$ \\
\hline Loops & Annotations from $\mathrm{Hi}-\mathrm{C}$ & $\begin{array}{l}\text { Mouse } \\
\mathrm{CH} 12-\mathrm{LX}\end{array}$ & 8 & $\begin{array}{l}\text { ftp://ftp.ncbi.nlm.nih.gov/geo/series/GSE63nnn/G } \\
\text { SE63525/suppl/GSE63525_CH12- } \\
\text { LX_HiCCUPS_looplist.txt.gz }\end{array}$ \\
\hline $\begin{array}{l}\text { CTCF M1 } \\
\text { motif }\end{array}$ & PWM for mouse & & 51 & $\begin{array}{l}\mathrm{ftp} / / \text { ftp.ebi.ac.uk/pub/databases/vertebrategeno } \\
\text { mics/FOG03/calls/CTCF_canonical_species }\end{array}$ \\
\hline \multirow[t]{2}{*}{$\begin{array}{l}\text { Lamin-B1 } \\
\text { DamID }\end{array}$} & $\begin{array}{l}\text { Normalized signal } \\
\text { Normalized signal } \\
\text { Normalized signal } \\
\text { Normalized signal }\end{array}$ & $\begin{array}{l}\text { Mouse ESCs } \\
\text { NPCs } \\
\text { Astrocytes } \\
\text { MEFs }\end{array}$ & 35 & $\begin{array}{l}\text { http://hgdownload.cse.ucsc.edu/goldenPath/mm9 } \\
\text { /database/laminB1_AC.txt.gz } \\
\text { http://hgdownload.cse.ucsc.edu/goldenPath/mm9 } \\
\text { /database/laminB1_EF.txt.gz } \\
\text { http://hgdownload.cse.ucsc.edu/goldenPath/mm9 } \\
\text { database/laminB1_ES.txt.gz } \\
\text { http://hgdownload.cse.ucsc.edu/goldenPath/mm9 } \\
\text { Ldatabase/laminB1_NP.txt.gz }\end{array}$ \\
\hline & HMM state calls & & 36 & $\begin{array}{l}\text { https://www.ncbi.nlm.nih.gov/geo/query/acc.cgi? } \\
\text { acc=GSE17051 }\end{array}$ \\
\hline $\begin{array}{l}\text { ChromH } \\
\text { MM }\end{array}$ & $\begin{array}{l}\text { Chromatin state } \\
\text { segmentation trained on } \\
\text { ENCODE mouse ChIP-seq } \\
\text { data }\end{array}$ & $\begin{array}{l}\text { Mouse } \\
\text { Liver, } \\
\text { Adult8wks }\end{array}$ & 50 & $\begin{array}{l}\text { https://github.com/gireeshkbogu/chromatin_state } \\
\text { s_chromHMM_mm9 }\end{array}$ \\
\hline $\begin{array}{l}\text { ENCODE } \\
\text { Histone } \\
\text { ChIP-seq }\end{array}$ & $\begin{array}{l}\text { ChIP-seq, } \\
\text { peak and bigwig files } \\
\text { Input } \\
\text { H3K } 27 \mathrm{ac} \\
\text { H3K } 27 \mathrm{me3} \\
\text { H3K } 4 \mathrm{me} 3 \\
\text { H3K4me1 } \\
\text { H3K9me3 } \\
\text { H3K36me3 } \\
\text { H3K } 79 \mathrm{me} 2\end{array}$ & $\begin{array}{l}\text { Mouse } \\
\text { Liver, } \\
\text { Adult8wks }\end{array}$ & $\begin{array}{l}42 \text { and } \\
\text { ENCODE }\end{array}$ & $\begin{array}{l}\text { http://hgdownload.cse.ucsc.edu/goldenPath/mm9 } \\
\text { LencodeDCC/wgEncodeLicrHistone/ }\end{array}$ \\
\hline $\begin{array}{l}\text { ENCODE } \\
\text { TFBS } \\
\text { ChIP-seq }\end{array}$ & $\begin{array}{l}\text { ChIP-seq, } \\
\text { Peak and bigwig files } \\
\text { Input } \\
\text { Polll } \\
\text { CTCF }\end{array}$ & $\begin{array}{l}\text { Mouse } \\
\text { Liver, } \\
\text { Adult8wks }\end{array}$ & $\begin{array}{l}42 \text { and } \\
\text { ENCODE }\end{array}$ & $\begin{array}{l}\text { http://hgdownload.cse.ucsc.edu/goldenPath } / \mathrm{mm} 9 \\
\text { LencodeDCC/wgEncodeLicrTfbs/ }\end{array}$ \\
\hline $\begin{array}{l}\text { ENCODE } \\
\text { DNase- } \\
\text { seq }\end{array}$ & $\begin{array}{l}\text { DNAse I HS, } \\
\text { bigwig }\end{array}$ & $\begin{array}{l}\text { Mouse } \\
\text { Liver, } \\
\text { Adult8wks }\end{array}$ & $\begin{array}{l}42 \text { and } \\
\text { ENCODE }\end{array}$ & $\begin{array}{l}\text { http://hgdownload.cse.ucsc.edu/goldenPath/mm9 } \\
\text { LencodeDCC/wgEncodeUwDnase/ }\end{array}$ \\
\hline $\begin{array}{l}\text { ENCODE } \\
\text { RNA-seq }\end{array}$ & $\begin{array}{l}\text { Poly-A RNA-seq, } \\
\text { bigwig } \\
\text { Poly-A RNA-seq, } \\
\text { bigwig }\end{array}$ & $\begin{array}{l}\text { Mouse } \\
\text { Liver, } \\
\text { Adult8wks } \\
\text { Mouse } \\
\text { Liver, } \\
\text { Adult8wks }\end{array}$ & $\begin{array}{l}42 \text { and } \\
\text { ENCODE }\end{array}$ & $\begin{array}{l}\text { http://hgdownload.cse.ucsc.edu/goldenPath/mm9 } \\
\text { LencodeDCC/wgEncodeUwRnaSeq/ } \\
\text { http://hgdownload.cse.ucsc.edu/goldenPath/mm9 } \\
\text { LencodeDCC/wgEncodeCshlLongRnaSeq/ }\end{array}$ \\
\hline
\end{tabular}




\section{Methods}

\section{Experimental procedures}

\section{Generation of Nipblflox/flox mice}

The Nipbl locus was targeted by introduction of two loxP sites flanking exon 18 via homologous recombination in E14 mouse embryonic stem cells. Individual ESC clones were screened for successful recombination by Southern blotting deploying two unique probes hybridizing 5' and 3' to the integration site, respectively. Cells of a successful clone were injected into mouse blastocysts and resultant chimera were bred to $\mathrm{C} 57 \mathrm{BL} / 6 \mathrm{~J}$ mice. Offspring were analyzed for successful germ line transmission by PCR and Southern blotting. Nipblfox/+ mice were maintained on C57BL/6J genetic background.

For deletion of the floxed exon, we used either a constitutive ubiquitous CRE-driver (Hprt::Cre ${ }^{39}$ ), a limb-specific CRE-driver (Prx1::Cre ${ }^{40}$ ) or an inducible, liver-specific Cre allele (Ttr-cre/Esr1 ${ }^{43}$.

Mice were genotyped by PCR using specifc primer pairs (details available on request).

All lines were maintained by breeding with C57B1/6J mice. Mouse experiments were conducted in accordance with the principles and guidelines in place at European Molecular Biology Laboratory, as defined and overseen by its Institutional Animal Care and Use Committee, in accordance with the European Directive 2010/63/EU.

\section{Generation and preparation of $\mathrm{Nipbl} /$ - adult primary hepatocytes.}

To inactivate $\mathrm{Nipbl}$ in adult mouse hepatocytes, $\mathrm{Nipblfox/+}^{\mathrm{f}}$ mice were crossed with mice carrying an inducible, liver-specific Cre allele (Ttr-cre/Esr1 ${ }^{43}$. Resultant double heterozygous mice were backcrossed to Nipblfox/fox. For experiments we used animals homozygous for the floxed Nipbl allele either carrying one or no copy of the inducible, liver-specific Cre allele as sample (Ttr-cre/Esr1+/wt; Nipblflox/flox $)$ or control (Ttr-cre/Esr1wt/wt ; Nipblflox/flox $)$, respectively.

12 week old mice were injected with $1 \mathrm{mg}$ Tamoxifen $(100 \mu \mathrm{l}$ of $10 \mathrm{mg} / \mathrm{ml}$ Tamoxifen in corn oil) on 5 consecutive days. After keeping these mice for another 5 days without injection, they were sacrificed and the hepatocytes were harvested. Until this time point, mice displayed no abnormal behavior, weight loss or any other obvious physiological changes. This was also the case, when we 
kept mice for additional 4 days without injection to test for any adverse effects immediately after our experimental time point.

The liver was dissected and the left lateral lobe was prepped for a two-step perfusion adapted from 44,45. First, the liver is perfused with an EDTA-containing buffer removing $\mathrm{Ca}^{2+}$ from the tissue. This weakens the integrity of the desmosome, which is then digested during the subsequent perfusion with a $\mathrm{Ca}^{2+}$ rich buffer containing collagenase. The freed hepatocytes were rinsed through a cell strainer and washed four times with ice-cold $\mathrm{Ca}^{2+}$ rich buffer without collagenase. For each wash the cells were spun at low centrifugal force $(60 \mathrm{~g}$ for $1 \mathrm{~min})$, to reduce non-mesenchymal cells and debris, hence, enriching intact hepatocytes in the sample. Part of each sample was fixed with 1\% PFA for 10 minutes at room temperature. Fixed and unfixed hepatocytes were aliquoted and frozen in liN $\mathrm{N}_{2}$ for later use.

\section{Nipbl RNA levels and activity}

Unfixed hepatocyte aliquots were thawed and RNA was prepared with Qiagen RNeasy Kit. cDNA was generated using NEB ProtoScript@ First Strand cDNA Synthesis Kit with random primer mix. RT-qPCR was performed with Applied SYBR Green PCR Master Mix and following primers: NipblqPCR_F TCCCCAGTATGACCCTGTTT, Nipbl-qPCR_R AGAACATTTAGCCCGTTTGG, Gapdh-qPCR_F CTCCCACTCTTCCACCTTCG， Gapdh-qPCR_R CCACCACCCTGTTGCTGTAG， RTqPCR_Pgk1_Fwd TGGTATACCTGCTGGCTGGATGG and RTqPCR_Pgk1_Rev GACCCACAGCCTCGGCATATTTC.

For Western blots unfixed hepatocyte aliquots were lysed and fractionated with a Subcellular Protein Fractionation Kit (ThermoFisher). The blots were probed with antibodies against cohesin subunits SA-1 and SMC1 (a courtesy of Ana Losada, CNIO, Madrid) and Topo II $\beta$ (611492, BD Biosciences) and Histone H2B (07-371, Millipore) as control for nuclear soluble and nuclear insoluble fractions, respectively.

\section{Immunohistochemistry on liver}

Slices of adult livers were collected and fixed in 4\% PFA overnight. After dehydration, the tissues were embedded in paraffin and sectioned at $6 \mu \mathrm{m}$. The sections were deparaffinized with xylene, rehydrated and antigens were retrieved by boiling in citrate buffer. The sections were blocked in 10\% FBS and incubated with primary antibodies ( $\alpha$ phospho-Histone H3, 06-570 Millipore; acleaved-caspase-3, \#9661 Cell Signaling) at $4^{\circ} \mathrm{C}$ overnight. Primary antibodies were detected with 
goat anti-rabbit IgG Alexa Fluor® 568 secondary antibody (A-11011, Invitrogen) and counter stained with DAPI. Images were acquired using confocal microscopy.

\section{RNA-seq libraries and sequencing}

RNA integrity was tested with Bioanalyzer (Agilent RNA Nano Kit) and ribosomal RNA was removed using Ribo-Zero rRNA Removal Kit (Illumina) prior to library preparation. Strand-specific libraries were prepared with NEBNext@ Ultra' ${ }^{\text {TM }}$ Directional RNA Library Prep Kit for Illumina®. After amplification and size selection with Agencourt AMPure XP beads (Beckmann Coulter) their size-distributions were determined with Bioanalyzer. Equimolar pools of libraries were sequenced with Illumina HiSeq2000 (50bp, single end). We retrieved on average 25 mio reads per sample, of which 19 mio reads were uniquely mapped to the reference genome (NCBI37/mm9).

\section{ChIP-seq libraries and sequencing}

Fixed aliquots of hepatocytes were hypotonically lysed and sonicated in 1\% SDS/TE. An aliquot of each sample was reverse cross-linked in order to determine chromatin concentration and sonication efficiency. $20 \mu \mathrm{g}$ chromatin per sample was diluted in RIPA and incubated with 1.5 $\mu \mathrm{g}$ of either $\alpha$ H3k4me3 antibody (C15410003-50, Diagenode) or $\alpha$ H3K27Ac antibody (ab4729, Abcam) at $4^{\circ} \mathrm{C}$, overnight. The antibodies were retrieved with Dynabeads (IgA, Invitrogen) and bound chromatin was washed and eluted. After reverse cross-linking, the amount of ChIPped and input DNA was determined with Qubit (Thermo Fisher). The libraries were prepared with NEBNext® ChIP-Seq Library Prep Kit for Illumina®. After amplification and size selection with E-Gel® SizeSelect ${ }^{\mathrm{TM}}$ (Thermo Fisher) their size-distributions were determined with Bioanalyzer. Equimolar pools of libraries were sequenced with Illumina HiSeq2000 (50bp, single end). We retrieved on average 20 mio reads per sample, of which 15 mio reads were uniquely mapped to the reference genome (NCBI37/mm9).

\section{Tethered Chromatin Capture (TCC)}

Roughly 100 mio fixed hepatocytes per sample were processed according to Kalhor et al. ${ }^{30}$ using HindIII. Libraries were PCR-amplified (12 cycles) and size selected with E-Gel® SizeSelect ${ }^{\mathrm{TM}}$ (Thermo Fisher). Equimolar pools of libraries were sequenced with Illumina HiSeq2000 (50bp, paired end). We retrieved between 100 and 150 mio paired reads per sample, of which $40 \%$ had both sides uniquely mapped to the reference genome (NCBI37/mm9). 


\section{Computational analysis}

\section{Preparation of Hi-C maps}

We mapped the sequence of $\mathrm{Hi}-\mathrm{C}$ molecules to reference mouse genome assembly mm 9 using Bowtie 2.2.8 and the iterative mapping strategy, as described in ${ }^{46}$ and implemented in the hiclib library for Python (publicly available at https://bitbucket.org/mirnylab/hiclib). Upon filtering PCR duplicates and reads mapped to multiple or zero locations, we aggregated the reads pairs into $20 \mathrm{~kb}$ and $100 \mathrm{~kb}$ genomic bins to produce Hi-C contact matrices. Low-coverage bins were then excluded from further analysis using the MAD-max (maximum allowed median absolute deviation) filter on genomic coverage, set to five median absolute deviations. To remove the short-range Hi-C artifacts unligated and self-ligated $\mathrm{Hi}-\mathrm{C}$ molecules - we removed contacts mapping to the same or adjacent genomic bins. The filtered $20 \mathrm{~kb}$ and $100 \mathrm{~kb}$ contacts matrices were then normalized using the iterative correction procedure (IC) ${ }^{46}$, such that the genome-wide sum of contact probability for each row/column equals 1.0. Observed/expected contact maps were obtained by dividing each diagonal of a contact map by its average value over non-filtered genomic bins.

The same procedure was used to analyse other existing Hi-C datasets on cohesin-depleted cells ${ }^{17-19}$.

\section{Compartment analysis via eigenvector decomposition}

The compartment structure of Hi-C maps was detected using a modified procedure from ${ }^{46}$. In short, compartments were quantified as the dominant eigenvector of the observed/expected $20 \mathrm{~kb}$ and $100 \mathrm{~kb}$ cis contacts maps upon subtraction of 1.0, as implemented in hiclib.

\section{Domain detection and loop coordinates}

To identify contact domains, we used a segmentation algorithm very similar to 47 , which divides the genome into domains in such a way as to maximize a global domain scoring function. We used two different scoring functions: one was the corner score function from ${ }^{8}$ and the other was based on network modularity ${ }^{48}$, which is a metric widely used to detect communities in networks. The modularity score for a domain spanning genomic bins $a$ to $b$ inclusively is given by

$$
S(a, b)=\sum_{i=a}^{b} \sum_{j=i}^{b} A_{i j}-\gamma N_{i j},
$$


where $A$ is the contact matrix and $N$ is the corresponding matrix of a penalizing background model. The resolution parameter controls the strength of the penalty and therefore the characteristic size of the domains identified.

By restricting the solution space to contiguous segmentations, both calculating domain scores and finding the highest scoring segmentation can be reduced to $\mathrm{O}\left(\mathrm{n}^{\wedge} 2\right)$ dynamic programming algorithms. Optimal segmentation, in particular, becomes the well-known max-sum algorithm on a weighted directed acyclic graph ${ }^{49}$. Furthermore, one can marginalize over the space of all possible segmentations to obtain a linear genomic track of boundary scores for a given domain scoring function, also in $\mathrm{O}\left(\mathrm{n}^{\wedge} 2\right)$ time. The implementation of these and related algorithms is provided in the lavaburst package (https://github.com/nezar-compbio/lavaburst).

The domain calls used in composite heatmaps were computed using the corner score on $20 \mathrm{~kb}$ resolution matrices. To robustly call insulating boundaries across different conditions, we exploit the multi-resolution nature of the modularity score and compute the average marginal boundary scores on $20 \mathrm{~kb} W T$ and $\Delta \mathrm{Nipbl}$ contact matrices sweeping over a range of gamma values to obtain a 1D boundary (i.e., insulation) track. Short intervals representing insulating loci were called by thresholding on the boundary score, and the common and unique loci to each condition were determined by interval intersection.

To characterize the structure of known loops in our data, we used the list of loops detected in Hi-C maps for CH12-LX mouse cell line in ${ }^{8}$.

\section{Analysis of epigenetic features}

To study the influence of the epigenetic chromatin state on genomic architecture, we used the ChromHMM genomic state annotation from ${ }^{50}$. Briefly, the authors trained the ChromHMM genomic segmentation model on H3K4me1, H3K4me3, H3K36me3, H3K27me3, H3K27ac, CTCF and RNA polymerase II ENCODE tracks for mouse liver. This model assigned to each genomic loci one of 15 possible epigenetic states. For our analyses, we further grouped these 15 states into three: Active (states 1-11, 14 and 15 characterized by presence of PolII), Repressed (states 10-12, characterized by high H3K27me3 and low H3K27ac emission probabilities) and Inert (state 13, lack of any signal).

To find the average footprint of a chromatin-bound CTCF, we detected all occurrences of the M1 CTCF motif 51 in the mm9 genome (filtered by p-value $>0.0001$ ), intersected with CTCF binding peaks in ENCODE adult mouse liver dataset ${ }^{52}$. This procedure yielded 27840 peaks. 
To study how lamina association affects genome compartmentalization, we used the dataset of Lamin-B1-binding loci from ${ }^{35}$ containing data from four mouse cell lines: embryonic stem cells (ESC), multipotent neural precursor cells (NPC), terminally differentiated astrocytes (AC) and embryonic fibroblasts (MEF). We then selected $100 \mathrm{~kb}$ genomic bins with more than 60 locations probed for lamin-B1 binding. We called the bins as constitutive LAD (cLAD) or non-LAD bins if $>90 \%$ of probed locations were bound to lamina or not bound to lamina across all four tested cell lines, respectively. Finally, bins were called facultative LADs if for more than $90 \%$ of probes showed lamina binding in some cell lines, but not in others.

\section{RNA-Seq}

We mapped the RNA-seq data to mm9 reference mouse genome assembly and GENCODE vM1 transcriptome ${ }^{53}$ using STAR v.2.5.0a ${ }^{54}$ and scripts from the ENCODE RNA-Seq pipeline [https://github.com/ENCODE-DCC/chip-seq-pipeline]. To obtain the tracks of local transcription, we aggregated the uniquely mapped reads into RPM-normalized bigWig files using the built-in STAR functionality. To find differentially expressed genes, we aggregated the read counts at the gene level using HTSeq ${ }^{55}$ with the "union" option and called DE genes with DESeq 256.

For identification of exogenic transcripts, we aligned reads on the mm9 genome using HISAT2 57 with the option to authorize splicing events. From these alignments, we count the number of reads in sliding windows of $1 \mathrm{~kb}$ (with a step of 600nt) taking each strand of the genome as separate. We use a gaussian mixture model ( 2 distributions) to find a cutoff value for readcounts differentiating "expressed" regions from noise. We choose a cutoff of 15 reads according to the best fitted gaussian distributions. To avoid overestimating the number of exogenic transcripts, we merged adjacent expressed windows (on the same strand) as composite transcripts. We used spliced reads to merge expressed windows that were not directly adjacent, but linked by spliced reads, with a cutoff of 7 splice reads. The resulting merged and linked windows defined the region corresponding to the exogenic transcription unit, and we took its 5' end (using the strand information, and after trimming the 5 'end of all nucleotides with no coverage) as transcriptional start site.

\section{ChIP-Seq}

We mapped the sequences obtained in ChIP-seq experiments following the ENCODE 3 pipeline [https://github.com/ENCODE-DCC/chip-seq-pipeline]. 


\section{Simulations of loop extrusion}

To investigate the impact of NIPBL depletion on TADs and loops in the context of loop extrusion, we performed simulations that couple the 1D dynamics of loop extrusion by LEFs with 3D polymer dynamics, as previously ${ }^{21}$. We then generated contact maps and calculated $\mathrm{P}(\mathrm{s})$, also as previously described.

We considered a system of 42 consecutive TADs of $400 \mathrm{~kb}$, where impermeable BEs were placed between each pair of neighboring TADs. We modeled the chromatin fiber as a series of $1 \mathrm{~kb}$ monomers $(\sim 6$ nucleosomes, $\sim 20 \mathrm{~nm})$, such that each TAD was 400 monomers. Polymer connectivity, stiffness, and excluded volume were implemented as previously described 21. Extruded loops held by LEFs were implemented by connecting the two monomers held by the two heads of each LEF with a harmonic bond, as previously ${ }^{21,58}$.

Three-dimensional polymer dynamics were implemented using OpenMM, a high-performance GPUassisted molecular dynamics API 59,60. Simulations were initialized from a compact polymer conformation, as described in [PMID: 25472862], created on a cubic lattice a box of the size (PBC box - 2). Prior to a block of simulations, LEF dynamics were advanced by 500,000 steps. To allow the polymer fiber to equilibrate with this set of LEF-imposed bonds, simulations were then advanced for 400 blocks of simulations. After that, 2000 blocks (ie. blocks of 1D+3D dynamics) of simulations were performed and their conformations were recorded. After that, LEF dynamics were advanced by 500,000 steps, and the process was repeated, until 5000 conformations for each parameter set were obtained.

For the control simulation, we considered LEFs with $200 \mathrm{~kb}$ processivity and $400 \mathrm{~kb}$ separation. To investigate the effect of depleting the amount of bound cohesin, we then increased LEF separation by 2-fold, 4-fold, and 8-fold. All simulations used a stiffness of 2, density of 0.2, 3D-to-1D-steps of 2500. To generate contact maps from simulated conformations, a capture radius of 6 was used.

For display, simulated contact maps were first binned using 10 monomer (40kb) bins. Then, the map was normalized such the average value of the first diagonal equals one, and $\log 10$ transformed. Finally, the color-scale was clipped to show 1.5 logarithmic orders of magnitude as its dynamic range. 
To calculate $P(s)$ plots from simulated data, a contact map with 1 monomer resolution was used. For each diagonal of the contact map, we determined which regions within 400-monomer TADs and which were between TADs. We then averaged the values in logarithmically-spaced bins of increasing distance with a step of 1.1. Similar to experimental data, $\mathrm{P}(\mathrm{s})$ curves were then vertically shifted such that $\mathrm{P}(\mathrm{s})$ at $10 \mathrm{~kb}$ was equal to 1 .

\section{Supplementary References}

39. Tang, S.-H. E., Silva, F. J., Tsark, W. M. K. \& Mann, J. R. A Cre/loxP-deleter transgenic line in mouse strain 129S1/SvImJ. Genesis 32, 199-202 (2002).

40. Logan, M. et al. Expression of Cre Recombinase in the developing mouse limb bud driven by a Prxl enhancer. Genesis 33, 77-80 (2002).

41. Kawauchi, S. et al. Multiple organ system defects and transcriptional dysregulation in the Nipbl(+/-) mouse, a model of Cornelia de Lange Syndrome. PLoS Genet 5, e1000650 (2009).

42. Shen, Y.et al. A map of the cis-regulatory sequences in the mouse genome. Nature 488, 116120 (2012).

43. Tannour-Louet, M., Porteu, A., Vaulont, S., Kahn, A. \& Vasseur-Cognet, M. A tamoxifeninducible chimeric Cre recombinase specifically effective in the fetal and adult mouse liver. Hepatology 35, 1072-1081 (2002).

44. Li, W.-C., Ralphs, K. L. \& Tosh, D. Isolation and culture of adult mouse hepatocytes. Methods Mol Biol 633, 185-196 (2010).

45. Gonçalves, L. A., Vigário, A. M. \& Penha-Gonçalves, C. Improved isolation of murine hepatocytes for in vitro malaria liver stage studies. Malar. J. 6, 169 (2007).

46. Imakaev, M. et al. Iterative correction of Hi-C data reveals hallmarks of chromosome organization. Nat Methods 9, 999-1003 (2012).

47. Filippova, D., Patro, R., Duggal, G. \& Kingsford, C. Identification of alternative topological domains in chromatin. Algorithms Mol Biol 9, 14 (2014).

48. Girvan, M. \& Newman, M. E. J. Community structure in social and biological networks. Proc Natl Acad Sci USA 99, 7821-7826 (2002).

49. Bishop, C. M. Pattern recognition. Machine Learning 128, (2006).

50. Bogu, G. K. et al. Chromatin and RNA Maps Reveal Regulatory Long Noncoding RNAs in Mouse. Mol Cell Biol 36, 809-819 (2015).

51. Schmidt, D. et al. Waves of Retrotransposon Expansion Remodel Genome Organization and CTCF Binding in Multiple Mammalian Lineages. Cell 148, 335-348 (2012).

52. Yue, F. et al. A comparative encyclopedia of DNA elements in the mouse genome. Nature 515, 355-364 (2014).

53. Harrow, J. et al. GENCODE: the reference human genome annotation for The ENCODE Project. Genome Res 22, 1760-1774 (2012).

54. Dobin, A. et al. STAR: ultrafast universal RNA-seq aligner. Bioinformatics 29, 15-21 (2013).

55. Anders, S., Pyl, P. T. \& Huber, W. HTSeq--a Python framework to work with high-throughput sequencing data. Bioinformatics 31, 166-169 (2015). 
56. Love, M. I., Huber, W. \& Anders, S. Moderated estimation of fold change and dispersion for RNA-seq data with DESeq2. Genome Biol 15, 550 (2014).

57. Kim, D., Langmead, B. \& Salzberg, S. L. HISAT: a fast spliced aligner with low memory requirements. Nat Methods 12, 357-360 (2015).

58. Goloborodko, A., Imakaev, M. V., Marko, J. F. \& Mirny, L. Compaction and segregation of sister chromatids via active loop extrusion. Elife 5, (2016).

59. Eastman, P. et al. Open MM 4: A Reusable, Extensible, Hardware Independent Library for High Performance Molecular Simulation. J Chem Theory Comput 9, 461-469 (2013).

60. Eastman, P. \& Pande, V. S. Efficient nonbonded interactions for molecular dynamics on a graphics processing unit. J Comput Chem 31, 1268-1272 (2010). 